Journal of Mathematical Analysis

ISSN: 2217-3412, URL: WWW.ILIRIAS.COM/JMA

Volume 12 Issue 5 (2021), Pages 1-27.

DOI:10.54379/JMA-2021-5-1

\title{
GENERALIZATION AND REFINEMENTS OF JENSEN INEQUALITY
}

\author{
FAIZA RUBAB, HIRA NABI AND ASIF R. KHAN
}

\begin{abstract}
We give generalizations and refinements of Jensen and JensenMercer inequalities by using weights which satisfy the conditions of Jensen and Jensen- Steffensen inequalities. We also give some refinements for discrete and integral version of generalized Jensen-Mercer inequality and shown to be an improvement of the upper bound for the Jensen's difference given in [32]. Applications of our work include new bounds for some important inequalities used in information theory, and generalizing the relations among means.
\end{abstract}

\section{INTRODUCTION}

Jensen's inequality for convex functions is one of the most celebrated inequalities in mathematics and statistics. Many other renowned inequalities can be obtained from it. For example, the important Arithmetic-Geometric inequality or the general inequality between means of orders $p$ and $q$, such as Hölder's and Minkowski's inequalities, are all consequences of Jensen's inequality for convex functions. Due to its fundamental importance, over the year it has been generalized to various context. There are given numerous variants, generalizations and refinements of Jensens inequalities for reference see [2], [3], [8], [9], [10], [11], [13], [14], [17], [18], [19], [20], [21], [22], [34], [36], [39], [40]. Throughout this paper, we assume $I$ is an interval in $\mathbb{R}$ and we assume $\mathbf{w}=\left(w_{1}, \ldots, w_{n}\right)$ be real $n$-tuple in $\mathbb{R}$ we define the notations

$$
W_{j}=\sum_{i=1}^{j} w_{i}, \quad j \in\{1, \ldots, n\} \text { and } \quad \bar{W}_{j}=\sum_{i=j}^{n} w_{i}=W_{n}-W_{j-1} .
$$

Now we state some preliminaries which are useful to prove our main results. Let us start with convex functions definition extracted from [33].

2010 Mathematics Subject Classification. 26D15, 39B99.

Key words and phrases. Jensen inequality; Jensen-Mercer inequality; Jensen-Steffensen inequality.

(C)2021 Ilirias Research Institute, Prishtinë, Kosovë.

The research of $2 n d$ and $3 r d$ author is supported by the Higher Education Commission of Pakistan under Indigenous Ph. D. Fellowship for 5000 Scholars, HEC, (phase-II) (PIN: 520 $143389-2$ PS6 - 17(50093221)).

Submitted October 16, 2020. Published July 27, 2021.

Communicated by Artion Kashuri. 
Definition 1.1. A function $\zeta: I \rightarrow \mathbb{R}$ is said to be convex if the inequality

$$
\zeta(\sigma \nu+(1-\sigma) \omega) \leq \sigma \zeta(\nu)+(1-\sigma) \zeta(\omega)
$$

holds for each $\nu, \omega \in I$ and $\sigma \in[0,1]$. If the inequality (1.1) is reversed, then $\zeta$ is said to be concave.

Remark 1.2. If $\alpha_{1}, \beta_{1}, \gamma_{1}$ are three points in $I$ such that $\alpha_{1}<\beta_{1}<\gamma_{1}$, then (1.1) is equivalent to (see $[33$, p. 2$]$

$$
\left|\begin{array}{lll}
\alpha_{1} & \zeta\left(\alpha_{1}\right) & 1 \\
\beta_{1} & \zeta\left(\beta_{1}\right) & 1 \\
\gamma_{1} & \zeta\left(\gamma_{1}\right) & 1
\end{array}\right| \geq 0
$$

which in turn is equivalent to

$$
\zeta\left(\beta_{1}\right) \leq \frac{\beta_{1}-\gamma_{1}}{\alpha_{1}-\gamma_{1}} \zeta\left(\alpha_{1}\right)+\frac{\alpha_{1}-\beta_{1}}{\alpha_{1}-\gamma_{1}} \zeta\left(\gamma_{1}\right)
$$

Theorem 1.3. Let $\zeta: I \rightarrow \mathbb{R}$ be a convex function. Let $n \geq 2, \mathbf{x}=\left(x_{1}, \ldots, x_{n}\right)$ be $n$-tuple in $I^{n}$ and $\mathbf{w}=\left(w_{1}, \ldots, w_{n}\right)$ be a positive $n$-tuple such that $W_{n}=\sum_{i=1}^{n} w_{i}$ $>0$. Then the well known Jensen's inequality

$$
\zeta\left(\frac{1}{W_{n}} \sum_{i=1}^{n} w_{i} x_{i}\right) \leq \frac{1}{W_{n}} \sum_{i=1}^{n} w_{i} \zeta\left(x_{i}\right) .
$$

holds (see [15] and [16] or [33, p. 43]).

In 2003, A. McD. Mercer [29] established a variant of Jensen inequality which is referred as "Jensen-Mercer inequality".

Theorem 1.4. Let $\zeta: I \rightarrow \mathbb{R}$ be a convex function and $\left[\eta_{1}, \theta_{1}\right] \subseteq I, \eta_{1}<\theta_{1}$. Let $\mathbf{x}=\left(x_{1}, \ldots, x_{n}\right)$ be $n$-tuple in $\left[\eta_{1}, \theta_{1}\right]^{n}$ and $\mathbf{w}=\left(w_{1}, \ldots, w_{n}\right)$ be a positive $n$-tuple. Then

$$
\zeta\left(\eta_{1}+\theta_{1}-\frac{1}{W_{n}} \sum_{i=1}^{n} w_{i} x_{i}\right) \leq \zeta\left(\eta_{1}\right)+\zeta\left(\theta_{1}\right)-\frac{1}{W_{n}} \sum_{i=1}^{n} w_{i} \zeta\left(x_{i}\right) .
$$

To prove the above variant A. McD. Mercer [29] first proved the following lemma.

Lemma 1.5. Let $\zeta: I \rightarrow \mathbb{R}$ be a convex function and $\left[\eta_{1}, \theta_{1}\right] \subseteq I, \eta_{1}<\theta_{1}$. Let $\mathbf{x}=\left(x_{1}, \ldots, x_{n}\right)$ be $n$-tuple in $\left[\eta_{1}, \theta_{1}\right]^{n}$, then

$$
\zeta\left(\eta_{1}+\theta_{1}-x_{i}\right) \leq \zeta\left(\eta_{1}\right)+\zeta\left(\theta_{1}\right)-\zeta\left(x_{i}\right), \quad i \in\{1, \ldots, n\} .
$$

Related to the Jensen's inequality, the following converse of Jensen's inequality was obtained (see [25] or [30, p. 9]).

Theorem 1.6. If $\zeta: I \longrightarrow \mathbb{R}$ is a convex function and $\left[\eta_{1}, \theta_{1}\right] \subseteq I, \eta_{1}<\theta_{1}$, $\mathbf{x}=\left(x_{1}, \ldots, x_{n}\right)$ be $n-$ tuple belongs to $\left[\eta_{1}, \theta_{1}\right]^{n}, \mathbf{w}=\left(w_{1}, \ldots, w_{n}\right)$ be a nonnegative $n$-tuple such that $W_{n}=\sum_{i=1}^{n} w_{i}>0$, then

$$
\frac{1}{W_{n}} \sum_{i=1}^{n} w_{i} \zeta\left(x_{i}\right) \leq \frac{\theta_{1}-\frac{1}{W_{n}} \sum_{i=1}^{n} w_{i} x_{i}}{\theta_{1}-\eta_{1}} \zeta\left(\eta_{1}\right)+\frac{\frac{1}{W_{n}} \sum_{i=1}^{n} w_{i} x_{i}-\eta_{1}}{\theta_{1}-\eta_{1}} \zeta\left(\theta_{1}\right)
$$

Equality holds in the above if and only if $x_{i}=\eta_{1}(i \in I \subset\{1, \ldots, n\})$ and $x_{i}=\theta_{1}(i \in\{1, \ldots, n\} \backslash I)$.

The following refinement related to converse of Jensen's inequality is obtained in $[4]$. 
Theorem 1.7. Under the assumptions of above Theorem 1.6 the following inequality holds

$$
\frac{1}{W_{n}} \sum_{i=1}^{n} w_{i} \zeta\left(x_{i}\right) \leq \frac{\theta_{1}-\check{x}}{\theta_{1}-\eta_{1}} \zeta\left(\eta_{1}\right)+\frac{\check{x}-\eta_{1}}{\theta_{1}-\eta_{1}} \zeta\left(\theta_{1}\right)-\left(\frac{1}{W_{n}} \sum_{i=1}^{n} w_{i}\left(\tilde{x}_{i}\right)\right) \delta_{\zeta}
$$

where, $\tilde{x}_{i}=\frac{1}{2}-\frac{1}{\theta_{1}-\eta_{1}}\left|x_{i}-\frac{\eta_{1}+\theta_{1}}{2}\right|, \delta_{\zeta}=\zeta\left(\eta_{1}\right)+\zeta\left(\theta_{1}\right)-2 \zeta\left(\frac{\eta_{1}+\theta_{1}}{2}\right)$ and $\check{x}=$ $\frac{1}{W_{n}} \sum_{i=1}^{n} w_{i} x_{i}$.

The condition "w is positive $n$-tuple" in Jensen inequality can be replaced by "w is a non-negative $n$-tuple" and $W_{n}>0$. It is reasonable to inquire whether the condition "w is a non-negative $n$-tuple" can be relaxed at the expense of restricting $\mathbf{x}$ more severely. Steffensen (see [37]) was the first to answer this query in Theorem 1.8 (see the proof of corresponding Theorem in [33]).

Theorem 1.8. If $\zeta: I \longrightarrow \mathbb{R}$ be a convex function and $\mathbf{x}=\left(x_{1}, \ldots, x_{n}\right)$ be a monotonic $n$-tuple in $I^{n}$ and $\mathbf{w}=\left(w_{1}, \ldots, w_{n}\right)$ be a real $n$-tuple such that,

$$
0 \leq W_{i} \leq W_{n}, \quad(1 \leq i \leq n-1), \quad W_{n}>0,
$$

then (1.3) still holds.

In 2005, Abramovich et. al. [1] and gave an interesting variant of Jensen-Steffensen inequality.

Theorem 1.9. Let $\zeta: I \longrightarrow \mathbb{R}$ be a convex function and $\left[\eta_{1}, \theta_{1}\right] \subseteq I, \eta_{1}<\theta_{1}$. If $\mathbf{x}=\left(x_{1}, \ldots, x_{n}\right)$ be a monotonic $n$-tuple in $\left[\eta_{1}, \theta_{1}\right]^{n}$ and $\mathbf{w}=\left(w_{1}, \ldots, w_{n}\right)$ be a real $n$-tuple such that $w_{i} \neq 0, i \in\{1, \ldots, n\}$ and (1.8) satisfied, then (1.4) still holds.

The maximum and minimum of any two numbers $y$ and $z$ can be defined as:

Definition 1.10. Let $X$ be a subset of $\mathbb{R}$ for every $y \in X$ the functions $|y|, y^{-}$ and $y^{+}$can be defined by

$$
\begin{gathered}
|y|(t)=|y(t)|, \quad \begin{array}{c}
y^{+}(t)=\max \{0, y(t)\}, \\
y^{+}+y^{-}=|y|,
\end{array} y^{+-y^{-}=y, \text { where }} \\
\min \{y, z\}=\frac{1}{2}(y+z-|y-z|), \\
\max \{y, z\}=\frac{1}{2}(y+z+|y-z|) .
\end{gathered}
$$

We need the following lemma given in [32] to obtain our main results related to the converse Jensen inequality.

Lemma 1.11. Let $\zeta$ be a convex function on $D_{\zeta}$. If $v_{1}, w_{1} \in D_{\zeta}$ (domain of the function $\zeta$ ) and $p, q \in[0,1]$ such that $p+q=1$. Then

$$
\begin{aligned}
\min \{p, q\}\left[\zeta\left(v_{1}\right)+\zeta\left(w_{1}\right)\right. & \left.-2 \zeta\left(\frac{v_{1}+w_{1}}{2}\right)\right] \\
\leq & p \zeta\left(v_{1}\right)+q \zeta\left(w_{1}\right)-\zeta\left(p v_{1}+q w_{1}\right) \\
\leq & \max \{p, q\}\left[\zeta\left(v_{1}\right)+\zeta\left(w_{1}\right)-2 \zeta\left(\frac{v_{1}+w_{1}}{2}\right)\right] .
\end{aligned}
$$

In paper [20] the following result is given: 
Theorem 1.12. Let $(\Omega, P, \mu)$ be a probability measure space and $f: \Omega \longrightarrow\left[\eta_{1}, \theta_{1}\right]$ is a measurable and integrable function. Then for any continuous convex function $\zeta:\left[\eta_{1}, \theta_{1}\right] \longrightarrow \mathbb{R}$, we have

$$
\zeta\left(\eta_{1}+\theta_{1}-\int_{\Omega} f d \mu\right) \leq \zeta\left(\eta_{1}\right)+\zeta\left(\theta_{1}\right)-\int_{\Omega} \zeta(f) d \mu
$$

Theorem 1.13. In [7], under the assumptions of above Theorem 1.12, the following series of inequalities are valid

$$
\begin{aligned}
\zeta\left(\eta_{1}+\theta_{1}-\int_{\Omega} f d \mu\right) & \leq \int_{\Omega} \zeta\left(\eta_{1}+\theta_{1}-f\right) d \mu \\
& \leq \frac{\theta_{1}-\int_{\Omega} f d \mu}{\theta_{1}-\eta_{1}} \zeta\left(\theta_{1}\right)+\frac{\int_{\Omega} f d \mu-\eta_{1}}{\theta_{1}-\eta_{1}} \zeta\left(\eta_{1}\right) \\
& \leq \zeta\left(\eta_{1}\right)+\zeta\left(\theta_{1}\right)-\int_{\Omega} \zeta(f) d \mu .
\end{aligned}
$$

In the present article, we generalize Jensen and Jensen-Mercer inequalities in different ways. We also give various refinements of generalized results. We organized the paper in the following manner: After some introduction and preliminaries, in Section 2, we generalize and refine Jensen inequality for the index set function under the conditions of Jensen-Steffensen's inequality. In Section 3, we generalize and refine Jensen-Mercer inequality. In Section 4, we generalize Jensen-Mercer inequality for the positive real weights and obtain some refinements. In Section 5 , we give an integral generalization of Jensen-Mercer inequality. The last Section 6 is based on some applications of the proposed results.

\section{Generalization and Refinements of Jensen Inequality For Index SET FunCtion}

In this section, we give generalization followed by refinement of Jensen inequality using index set functions.

Let $J$ be a finite nonempty subset of positive integers and let $\zeta: I \longrightarrow \mathbb{R}$ be a function. Let $x=\left\{x_{i}\right\}_{i \in J}$ be a real monotonic sequence such that $x_{i} \in I$ for all $i \in J$ and $w=\left\{w_{i}\right\}_{i \in J}$ be a real sequence such that $\frac{1}{W_{J}} \sum_{i \in J} w_{i} x_{i} \in I$, where $W_{J}=\sum_{i \in J} w_{i}$. Let us define the index set function $F$ as

$$
F(J)=W_{J}\left[\zeta\left(\frac{1}{W_{J}} \sum_{i \in J} w_{i} x_{i}\right)-\frac{1}{W_{J}} \sum_{i \in J} w_{i} \zeta\left(x_{i}\right)\right]
$$

Then the following result is valid.

Theorem 2.1. Let $\zeta: I \rightarrow \mathbb{R}$ be a function. Let $J$ and $K$ be finite non-empty subsets of positive integers such that $J \cap K=\phi$ and $J \cup K=\{1, \ldots, n\}$. Let $x=\left\{x_{i}\right\}_{i \in J \cup K}$ be a real monotonic sequence such that $x_{i} \in I$ for all $i \in J \cup K$ and $w=\left\{w_{i}\right\}_{i \in J \cup K}$ be a real sequence such that $\frac{1}{W_{S}} \sum_{i \in S} w_{i} x_{i} \in I(S=J, K, J \cup K)$ and $0<W_{S}<W_{J \cup K}$, where equality holds for $S=J \cup K$. If $\zeta$ is convex on $I$, then $F$ is subadditive, i.e.,

$$
F(J \cup K) \leq F(J)+F(K)
$$


Proof.

$$
\begin{aligned}
& F(J \cup K)=W_{J \cup K}\left[\zeta\left(\frac{1}{W_{J \cup K}} \sum_{i \in J \cup K} w_{i} x_{i}\right)-\frac{1}{W_{J \cup K}} \sum_{i \in J \cup K} w_{i} \zeta\left(x_{i}\right)\right] \\
& =W_{J \cup K} \zeta\left(\frac{1}{W_{J \cup K}} \sum_{i \in J \cup K} w_{i} x_{i}\right)-\sum_{i \in J \cup K} w_{i} \zeta\left(x_{i}\right),
\end{aligned}
$$

while convexity of $\zeta$ and Jensen-Steffensen inequality yeids

$$
\begin{aligned}
& \zeta\left(\frac{1}{W_{J \cup K}} \sum_{i \in J \cup K} w_{i} x_{i}\right)=\zeta\left(\frac{1}{W_{J \cup K}}\left(\sum_{i \in J} w_{i} x_{i}+\sum_{i \in K} w_{i} x_{i}\right)\right) \\
& =\zeta\left(\frac{W_{J}}{W_{J \cup K}}\left(\frac{1}{W_{J}} \sum_{i \in J} w_{i} x_{i}\right)+\frac{W_{K}}{W_{J \cup K}}\left(\frac{1}{W_{K}} \sum_{i \in K} w_{i} x_{i}\right)\right) \\
& \leq \frac{W_{J}}{W_{J \cup K}} \zeta\left(\frac{1}{W_{J}} \sum_{i \in J} w_{i} x_{i}\right)+\frac{W_{K}}{W_{J \cup K}} \zeta\left(\frac{1}{W_{K}} \sum_{i \in K} w_{i} x_{i}\right) .
\end{aligned}
$$

Finally combining (2.2) and inequality (2.3) we get

$$
\begin{aligned}
& F(J \cup K) \\
& \leq W_{J} \zeta\left(\frac{1}{W_{J}} \sum_{i \in J} w_{i} x_{i}\right)+W_{K} \zeta\left(\frac{1}{W_{K}} \sum_{i \in K} w_{i} x_{i}\right)-\sum_{i \in J \cup K} w_{i} \zeta\left(x_{i}\right) \\
& =W_{J} \zeta\left(\frac{1}{W_{J}} \sum_{i \in J} w_{i} x_{i}\right)+W_{K} \zeta\left(\frac{1}{W_{K}} \sum_{i \in K} w_{i} x_{i}\right)-\left(\sum_{i \in J} w_{i}+\sum_{i \in K} w_{i}\right) \zeta\left(x_{i}\right) .
\end{aligned}
$$

After rearrangement we get

$$
\begin{aligned}
& F(J \cup K) \leq W_{J}\left(\zeta\left(\frac{1}{W_{J}} \sum_{i \in J} w_{i} x_{i}\right)-\frac{1}{W_{J}} \sum_{i \in J} w_{i} \zeta\left(x_{i}\right)\right)+ \\
& W_{K}\left(\zeta\left(\frac{1}{W_{K}} \sum_{i \in K} w_{i} x_{i}\right)-\frac{1}{W_{K}} \sum_{i \in K} w_{i} \zeta\left(x_{i}\right)\right) \\
& =F(J)+F(K),
\end{aligned}
$$

which shows that Jensen-Steffensen functional is subadditive.

Remark 2.2. From Theorem 2.1 as $J \cup K=\{1, \ldots, n\}$ we can conclude by using Theorem 1.8. that $F(J \cup K) \leq 0$.

Remark 2.3. Theorem 2.1. captures Theorem 1 of [38] as a special case.

Now we state a corollary for $k$ disjoint nonempty subsets of positive integers.

Corollary 2.4. Let $\zeta: I \longrightarrow \mathbb{R}$ be a function. Let $J_{1}, \ldots, J_{k}$ be finite non-empty subsets of positive integers such that $J_{i} \cap J_{j}=\phi$, for all $i \neq j \in\{1, \ldots, k\}$. Let $x=\left\{x_{i}\right\}_{i \in \cup_{j=1}^{k} J_{j}}$ be a real monotonic sequence such that $x_{i} \in I$ for all $i \in$ $\cup_{j=1}^{k} J_{j}$ and $w=\left\{w_{i}\right\}_{i \in \cup_{j=1}^{k} J_{j}}$ be a real sequence such that $\frac{1}{W_{S}} \sum_{i \in S} w_{i} x_{i} \in I$ 
$\left(S \in\left\{J_{1}, \ldots, J_{k}, \cup_{j=1}^{l} J_{j}\right\}, \quad l \in\{2, \ldots, k\}\right)$, and $0<W_{S}<W_{\cup_{j=1}^{k} J_{j}}$ where equality holds for $S=\cup_{j=1}^{l} J_{j}$. If $\zeta$ is convex on $I$, then

$$
F\left(\bigcup_{j=1}^{k} J_{j}\right) \leq \sum_{j=1}^{k} F\left(J_{j}\right) .
$$

Proof. This is a simple consequence of the fact,

$$
F\left(\bigcup_{j=1}^{k+1} J_{j}\right) \leq F\left(\bigcup_{j=1}^{k} J_{j}\right)+F\left(J_{k+1}\right) \leq \sum_{j=1}^{k} F\left(J_{j}\right)+F\left(J_{k+1}\right)=\sum_{j=1}^{k+1} F\left(J_{j}\right) .
$$

We get last inequality by index hypothesis which is

$$
F\left(\bigcup_{j=1}^{k} J_{j}\right) \leq \sum_{j=1}^{k} F\left(J_{j}\right)
$$

Now we state another corollary of Theorem 2.1.

Corollary 2.5. Let $\zeta: I \longrightarrow \mathbb{R}$ be a function. Let $J_{k}=\{1, \ldots, k\}$ for $k \in$ $\{1, \ldots, n\}$. Let $x=\left\{x_{i}\right\}_{i \in J_{n}}$ be a real monotonic sequence such that $x_{i} \in I$ for all $i \in J_{n}$ and $w=\left\{w_{i}\right\}_{i \in J_{n}}$ be a real sequence such that $w_{i} \neq 0$ for $i \in J_{n}$. Further suppose $\frac{1}{W_{S}} \sum_{i \in S} w_{i} x_{i} \in I \quad\left(S \in\left\{J_{1}, \ldots, J_{n}\right\}\right)$ and $0<W_{S}<W_{J_{n}}$, where equality holds for $S=J_{n}$. If $\zeta$ is convex on $I$, then we have

$$
F\left(J_{n}\right) \leq F\left(J_{n-1}\right) \leq \cdots \leq F\left(J_{2}\right) \leq 0 .
$$

Proof. As we have

$$
F(\{k\})=w_{k}\left(\zeta\left(\frac{1}{w_{k}} w_{k} x_{k}\right)-\frac{1}{w_{k}} w_{k} \zeta\left(x_{k}\right)\right)=0 .
$$

Now, by Theorem 2.1 for all $k \in\{2, \ldots, n\}$, we have

$$
F\left(J_{k}\right)=F\left(J_{k-1} \cup\{k\}\right) \leq F\left(J_{k-1}\right)+F(\{k\}),
$$

by using (2.6) we have

$$
F\left(J_{k}\right) \leq F\left(J_{k-1}\right)
$$

for all $k \in\{2, \ldots, n\}$. The last inequality in (2.5) is obvious as

$$
F\left(J_{2}\right)=F(\{1\} \cup\{2\}) \leq F(\{1\})+F(\{2\})=0 .
$$

The special case of Corollary 2.5 can be found in [30, p. 7] which may be stated as follows.

Corollary 2.6. Let $\zeta: I \longrightarrow \mathbb{R}$ be a function. Let $J_{k}=\{1, \ldots, k\}$ for $k \in$ $\{1, \ldots, n\}$. Let $x=\left\{x_{i}\right\}_{i \in J_{n}}$ and $w=\left\{w_{i}\right\}_{i \in J_{n}}$ be a real sequence such that $x_{i} \in I$, and $w_{i} \geq 0$ for all $i \in J_{n}$. If $\zeta$ is convex on $I$, then we have (2.5) and

$$
F\left(J_{n}\right) \leq \min _{1 \leq i<j<\leq n}\left\{\left(w_{i}+w_{j}\right) \zeta\left(\frac{w_{i} x_{i}+w_{j} x_{j}}{w_{i}+w_{j}}\right)-w_{i} \zeta\left(x_{i}\right)-w_{j} \zeta\left(x_{j}\right)\right\}
$$


Remark 2.7. Analogues assertions can be formulated for concave functions in Theorem 2.1, Corollary 2.4, Corollary 2.5 and Corollary 2.6 applying the fact that $\zeta$ is concave iff $-\zeta$ is convex.

\section{Generalization and Refinements of Jensen-Mercer Inequality for INDEX SET FUNCTION}

In this section, we give generalization followed by refinement of Jensen-Mercer inequality for index set functions. Let $J$ be a finite nonempty subset of positive integers and let $\zeta: I \longrightarrow \mathbb{R}$ be a function and $\left[\eta_{1}, \theta_{1}\right] \subseteq I, \eta_{1}<\theta_{1}$. Let $x=$ $\left\{x_{i}\right\}_{i \in J}$ be a real monotonic sequence such that $x_{i} \in\left[\eta_{1}, \theta_{1}\right]$ for all $i \in J$, and $w=\left\{w_{i}\right\}_{i \in J}$ be a real sequence such that $w_{i} \neq 0$ and $\frac{1}{W_{J}} \sum_{i \in J} w_{i} x_{i} \in\left[\eta_{1}, \theta_{1}\right]$, where $W_{J}=\sum_{i \in J} w_{i}$. Let us define the index set function $\widetilde{F}$ as

$$
\widetilde{F}(J)=W_{J}\left[\zeta\left(\eta_{1}\right)+\zeta\left(\theta_{1}\right)-\frac{1}{W_{J}} \sum_{i \in J} w_{i} \zeta\left(x_{i}\right)-\zeta\left(\eta_{1}+\theta_{1}-\frac{1}{W_{J}} \sum_{i \in J} w_{i} x_{i}\right)\right] .
$$

Then the following result is valid.

Theorem 3.1. Let $\zeta: I \rightarrow \mathbb{R}$ be a function and $\left[\eta_{1}, \theta_{1}\right] \subseteq I, \eta_{1}<\theta_{1}$. Let $J$ and $K$ be finite non-empty subsets of positive integers such that $J \cap K=\phi$ and $J \cup K=\{1, \ldots, n\}$. Let $x=\left\{x_{i}\right\}_{i \in J \cup K}$ be a real monotonic sequence such that $x_{i} \in\left[\eta_{1}, \theta_{1}\right]$ for all $i \in J \cup K$, and $w=\left\{w_{i}\right\}_{i \in J \cup K}$ be a real sequence such that $w_{i} \neq 0$. Further suppose $\frac{1}{W_{S}} \sum_{i \in S} w_{i} x_{i} \in\left[\eta_{1}, \theta_{1}\right] \quad(S=J, K, J \cup K)$ and $0<W_{S}<W_{J \cup K}$, where equality holds for $S=J \cup K$. If $\zeta$ is convex on $I$, then $\widetilde{F}$ is superadditive, i.e.,

$$
\widetilde{F}(J \cup K) \geq \widetilde{F}(J)+\widetilde{F}(K)
$$

Proof.

$$
\begin{aligned}
& \widetilde{F}(J \cup K) \\
& =W_{J \cup K}\left[\zeta\left(\eta_{1}\right)+\zeta\left(\theta_{1}\right)-\frac{1}{W_{J \cup K}} \sum_{i \in J \cup K} w_{i} \zeta\left(x_{i}\right)-\zeta\left(\eta_{1}+\theta_{1}-\frac{1}{W_{J \cup K}} \sum_{i \in J \cup K} w_{i} x_{i}\right)\right] \\
& =W_{J \cup K}\left(\zeta\left(\eta_{1}\right)+\zeta\left(\theta_{1}\right)-\frac{1}{W_{J \cup K}} \sum_{i \in J \cup K} w_{i} \zeta\left(x_{i}\right)\right) \\
& -W_{J \cup K} \zeta\left(\eta_{1}+\theta_{1}-\frac{1}{W_{J \cup K}} \sum_{i \in J \cup K} w_{i} x_{i}\right)
\end{aligned}
$$


while convexity of $\zeta$ and Jensen-Steffensen inequality yields

$$
\begin{aligned}
& \zeta\left(\eta_{1}+\theta_{1}-\frac{1}{W_{J \cup K}} \sum_{i \in J \cup K} w_{i} x_{i}\right) \\
& =\zeta\left(\frac{1}{W_{J \cup K}}\left[W_{J \cup K}\left(\eta_{1}+\theta_{1}\right)-\sum_{i \in J \cup K} w_{i} x_{i}\right]\right) \\
& =\zeta\left(\frac{1}{W_{J \cup K}}\left[\left(\sum_{i \in J} w_{i}+\sum_{i \in K} w_{i}\right)\left(\eta_{1}+\theta_{1}\right)-\left(\sum_{i \in J} w_{i} x_{i}+\sum_{i \in K} w_{i} x_{i}\right)\right]\right) \\
& =\zeta\left(\frac{W_{J}}{W_{J \cup K}}\left(\eta_{1}+\theta_{1}-\frac{1}{W_{J}} \sum_{i \in J} w_{i} x_{i}\right)+\frac{W_{K}}{W_{J \cup K}}\left(\eta_{1}+\theta_{1}-\frac{1}{W_{K}} \sum_{i \in K} w_{i} x_{i}\right)\right) \\
& \leq \frac{W_{J}}{W_{J \cup K}} \zeta\left(\eta_{1}+\theta_{1}-\frac{1}{W_{J}} \sum_{i \in J} w_{i} x_{i}\right)+\frac{W_{K}}{W_{J \cup K}} \zeta\left(\eta_{1}+\theta_{1}-\frac{1}{W_{K}} \sum_{i \in K} w_{i} x_{i}\right) .
\end{aligned}
$$

Finally combining (3.3) and inequality (3.4) we get

$$
\begin{aligned}
& \widetilde{F}(J \cup K) \geq W_{J \cup K}\left(\zeta\left(\eta_{1}\right)+\zeta\left(\theta_{1}\right)-\frac{1}{W_{J \cup K}} \sum_{i \in J \cup K} w_{i} \zeta\left(x_{i}\right)\right) \\
& -W_{J \cup K}\left[\frac{W_{J}}{W_{J \cup K}} \zeta\left(\eta_{1}+\theta_{1}-\frac{1}{W_{J}} \sum_{i \in J} w_{i} x_{i}\right)+\frac{W_{K}}{W_{J \cup K}} \zeta\left(\eta_{1}+\theta_{1}-\frac{1}{W_{K}} \sum_{i \in K} w_{i} x_{i}\right)\right] \\
& =W_{J \cup K}\left(\zeta\left(\eta_{1}\right)+\zeta\left(\theta_{1}\right)\right)-\sum_{i \in J \cup K} w_{i} \zeta\left(x_{i}\right) \\
& -W_{J \cup K}\left[\frac{W_{J}}{W_{J \cup K}} \zeta\left(\eta_{1}+\theta_{1}-\frac{1}{W_{J}} \sum_{i \in J} w_{i} x_{i}\right)+\frac{W_{K}}{W_{J \cup K}} \zeta\left(\eta_{1}+\theta_{1}-\frac{1}{W_{K}} \sum_{i \in K} w_{i} x_{i}\right)\right] \\
& =\left(\sum_{i \in J} w_{i}+\sum_{i \in K} w_{i}\right)\left(\zeta\left(\eta_{1}\right)+\zeta\left(\theta_{1}\right)\right)-\left(\sum_{i \in J} w_{i}+\sum_{i \in K} w_{i}\right) \zeta\left(x_{i}\right) \\
& -W_{J} \zeta\left(\eta_{1}+\theta_{1}-\frac{1}{W_{J}} \sum_{i \in J} w_{i} x_{i}\right)-W_{K} \zeta\left(\eta_{1}+\theta_{1}-\frac{1}{W_{K}} \sum_{i \in K} w_{i} x_{i}\right) .
\end{aligned}
$$

After rearrangement we get

$$
\begin{aligned}
& \widetilde{F}(J \cup K) \geq \\
& W_{J}\left(\zeta\left(\eta_{1}\right)+\zeta\left(\theta_{1}\right)-\frac{1}{W_{J}} \sum_{i \in J} w_{i} \zeta\left(x_{i}\right)\right)+W_{K}\left(\zeta\left(\eta_{1}\right)+\zeta\left(\theta_{1}\right)-\frac{1}{W_{K}} \sum_{i \in K} w_{i} \zeta\left(x_{i}\right)\right) \\
& -W_{J} \zeta\left(\eta_{1}+\theta_{1}-\frac{1}{W_{J}} \sum_{i \in J} w_{i} x_{i}\right)-W_{K} \zeta\left(\eta_{1}+\theta_{1}-\frac{1}{W_{K}} \sum_{i \in K} w_{i} x_{i}\right) .
\end{aligned}
$$


Finally we have

$$
\begin{aligned}
& \widetilde{F}(J \cup K) \geq \\
& W_{J}\left(\zeta\left(\eta_{1}\right)+\zeta\left(\theta_{1}\right)-\frac{1}{W_{J}} \sum_{i \in J} w_{i} \zeta\left(x_{i}\right)-\zeta\left(\eta_{1}+\theta_{1}-\frac{1}{W_{J}} \sum_{i \in J} w_{i} x_{i}\right)\right) \\
& +W_{K}\left(\zeta\left(\eta_{1}\right)+\zeta\left(\theta_{1}\right)-\frac{1}{W_{K}} \sum_{i \in K} w_{i} \zeta\left(x_{i}\right)-\zeta\left(\eta_{1}+\theta_{1}-\frac{1}{W_{K}} \sum_{i \in K} w_{i} x_{i}\right)\right) \\
& =\widetilde{F}(J)+\widetilde{F}(K),
\end{aligned}
$$

which shows Jensen-Steffensen functional is superadditive.

Remark 3.2. From Theorem 3.1. as $J \cup K=\{1, \ldots, n\}$ we can conclude by using Theorem 1.9. that $\widetilde{F}(J \cup K) \geq 0$.

Remark 3.3. Theorem 3.1. captures Theorem 2.3. of [28] as a special case.

Now we state a corollary for $k$ disjoint nonempty subsets of positive integers.

Corollary 3.4. Let $\zeta: I \longrightarrow \mathbb{R}$ be a function and $\left[\eta_{1}, \theta_{1}\right] \subseteq I, \eta_{1}<\theta_{1}$. Let $J_{1}, \ldots, J_{k}$ be finite non-empty subsets of positive integers such that $J_{i} \cap J_{j}=\phi$, for all $i \neq j \in\{1, \ldots, k\}$. Let $x=\left\{x_{i}\right\}_{i \in \cup_{j=1}^{k} J_{j}}$ be a real monotonic sequence such that $x_{i} \in\left[\eta_{1}, \theta_{1}\right]$ for all $i \in \cup_{j=1}^{k} J_{j}$, and $w=\left\{w_{i}\right\}_{i \in \cup_{j=1}^{k} J_{j}}$ be a real sequence such that $w_{i} \neq 0$ for all $i \in \cup_{j=1}^{k} J_{j}$. Further suppose $\frac{1}{W_{S}} \sum_{i \in S} w_{i} x_{i} \in\left[\eta_{1}, \theta_{1}\right]$ $\left(S \in\left\{J_{1}, \ldots, J_{k}, \cup_{j=1}^{l} J_{j}\right\}, l \in\{2, \ldots, k\}\right)$ and $0<W_{S}<W_{\cup_{j=1}^{k} J_{j}}$, where equality holds for $S=\cup_{j=1}^{k} J_{j}$. If $\zeta$ is convex on $I$, then

$$
\widetilde{F}\left(\bigcup_{j=1}^{k} J_{j}\right) \geq \sum_{j=1}^{k} \widetilde{F}\left(J_{j}\right) .
$$

Proof. The proof follows the same argument as in proof of Corollary 2.4.

Now we state another corollary of Theorem 3.1.

Corollary 3.5. Let $\zeta: I \longrightarrow \mathbb{R}$ be a function and $\left[\eta_{1}, \theta_{1}\right] \subseteq I, \eta_{1}<\theta_{1}$. Let $J_{k}=\{1, \ldots, k\}$ for $k \in\{1, \ldots, n\}$. Let $x=\left\{x_{i}\right\}_{i \in J_{n}}$ be a real monotonic sequence such that $x_{i} \in\left[\eta_{1}, \theta_{1}\right]$ for all $i \in J_{n}$ and $w=\left\{w_{i}\right\}_{i \in J_{n}}$ be a real sequence such that $w_{i} \neq 0$ for $i \in J_{n}$. Further suppose $\frac{1}{W_{S}} \sum_{i \in S} w_{i} x_{i} \in\left[\eta_{1}, \theta_{1}\right] \quad\left(S \in\left\{J_{1}, \ldots, J_{n}\right\}\right)$ and $0<W_{S}<W_{J_{n}}$, where equality holds for $S=J_{n}$. If $\zeta$ is convex on $I$, then we have

$$
\widetilde{F}\left(J_{n}\right) \geq \widetilde{F}\left(J_{n-1}\right) .
$$

Proof. Since $\zeta$ is convex function hence by using Lemma 1.5 for $x_{n} \in\left[\eta_{1}, \theta_{1}\right]$ we get

$$
\zeta\left(\eta_{1}+\theta_{1}-x_{n}\right) \leq \zeta\left(\eta_{1}\right)+\zeta\left(\theta_{1}\right)-\zeta\left(x_{n}\right) .
$$

From given condition we have $0<W_{J_{n-1}}<W_{J_{n}}$ which implies $0<w_{n}=W_{J_{n}}-$ $W_{J_{n-1}}$. So by applying (3.6) we have

$$
\widetilde{F}(\{n\})=w_{n}\left(\zeta\left(\eta_{1}\right)+\zeta\left(\theta_{1}\right)-\zeta\left(x_{n}\right)-\zeta\left(\eta_{1}+\theta_{1}-x_{n}\right)\right) \geq 0 .
$$


As

$$
\widetilde{F}\left(J_{n}\right)=\widetilde{F}\left(J_{n-1} \cup\{n\}\right) .
$$

Since, $J_{n-1} \cap\{n\}=\phi$, hence by Theorem 3.1 and then by using inequality (3.7) we get

$$
\widetilde{F}\left(J_{n}\right)=\widetilde{F}\left(J_{n-1} \cup\{n\}\right) \geq \widetilde{F}\left(J_{n-1}\right)+\widetilde{F}(\{n\}) \geq \widetilde{F}\left(J_{n-1}\right) .
$$

Remark 3.6. Analogues assertions can be formulated for concave functions in Theorem 3.1., Corollary 3.4. and Corollary 3.5., applying the fact that $\zeta$ is concave iff $-\zeta$ is convex.

\section{Refinements of Discrete Jensen-Mercer Inequality}

The following lemma is proved on the basis of the basic property of convexity as mentioned in the remark 1.2.

Lemma 4.1. Let $\zeta: I \longrightarrow \mathbb{R}$ be a convex function and $\left[\eta_{1}, \theta_{1}\right] \subseteq I, \mathbf{x}=\left(x_{1}, \ldots, x_{n}\right) \in$ $\left[\eta_{1}, \theta_{1}\right]^{n}$ such that $\left(\eta_{1}<\theta_{1}\right), \eta_{1} \leq x_{i} \leq \theta_{1}$ and $\mathbf{w}=\left(w_{1}, \ldots, w_{n}\right)$ be a positive $n$-tuple such that $W_{n}=\sum_{i=1}^{n} w_{i}>0$. We have,

$\frac{1}{W_{n}} \sum_{i=1}^{n} w_{i} \zeta\left(\eta_{1}+\theta_{1}-x_{i}\right) \leq \frac{\theta_{1}-\frac{1}{W_{n}} \sum_{i=1}^{n} w_{i} x_{i}}{\theta_{1}-\eta_{1}} \zeta\left(\theta_{1}\right)+\frac{\frac{1}{W_{n}} \sum_{i=1}^{n} w_{i} x_{i}-\eta_{1}}{\theta_{1}-\eta_{1}} \zeta\left(\eta_{1}\right)$.

Proof. Let $i \in\{1, \ldots, n\}$ be an arbitrary fixed positive integer. If $\eta_{1}, x_{i}, \theta_{1}$ are three points in $\left[\eta_{1}, \theta_{1}\right] \subseteq I$ such that $\eta_{1} \leq x_{i} \leq \theta_{1}$ and $\zeta$ is a convex function, then according to the definition of convex function, which is equivalent to the following as given in remark 1.2.

$$
\left|\begin{array}{ccc}
\eta_{1} & \zeta\left(\eta_{1}\right) & 1 \\
\eta_{1}+\theta_{1}-x_{i} & \zeta\left(\eta_{1}+\theta_{1}-x_{i}\right) & 1 \\
\theta_{1} & \zeta\left(\theta_{1}\right) & 1
\end{array}\right| \geq 0
$$

gives us

$$
\zeta\left(\eta_{1}+\theta_{1}-x_{i}\right)\left(\eta_{1}-\theta_{1}\right)+\zeta\left(\theta_{1}\right)\left(\theta_{1}-x_{i}\right)+\zeta\left(\eta_{1}\right)\left(x_{i}-\eta_{1}\right) \geq 0 .
$$

which is equivalent to,

$$
\zeta\left(\eta_{1}+\theta_{1}-x_{i}\right) \leq \frac{\theta_{1}-x_{i}}{\theta_{1}-\eta_{1}} \zeta\left(\theta_{1}\right)+\frac{x_{i}-\eta_{1}}{\theta_{1}-\eta_{1}} \zeta\left(\eta_{1}\right) .
$$

now multiplying by $w_{i}$ and taking sum over $i$ from 1 to $n$, then after dividing by $W_{n}$, we finally get

$$
\frac{1}{W_{n}} \sum_{i=1}^{n} w_{i} \zeta\left(\eta_{1}+\theta_{1}-x_{i}\right) \leq \frac{\theta_{1}-\frac{1}{W_{n}} \sum_{i=1}^{n} w_{i} x_{i}}{\theta_{1}-\eta_{1}} \zeta\left(\theta_{1}\right)+\frac{\frac{1}{W_{n}} \sum_{i=1}^{n} w_{i} x_{i}-\eta_{1}}{\theta_{1}-\eta_{1}} \zeta\left(\eta_{1}\right) .
$$

Here is another proof of the above lemma, since $x_{i} \in\left[\eta_{1}, \theta_{1}\right] \forall i \in\{1,2, \ldots, n\}$ therefore $\eta_{1}+\theta_{1}-x_{i} \in\left[\eta_{1}, \theta_{1}\right]$, if we replace $x_{i}$ by $\eta_{1}+\theta_{1}-x_{i}$ in (1.6) we get the required result (4.1).

Remark 4.2. Note that if the function $\zeta$ is concave, inequality (4.1) is reversed.ss 
Theorem 4.3. Under the assumptions of Theorem 1.4., the following series of inequalities is valid

$$
\begin{aligned}
\zeta\left(\eta_{1}+\theta_{1}-\right. & \left.\frac{1}{W_{n}} \sum_{i=1}^{n} w_{i} x_{i}\right) \leq \frac{1}{W_{n}} \sum_{i=1}^{n} w_{i} \zeta\left(\eta_{1}+\theta_{1}-x_{i}\right) \\
\leq \frac{\theta_{1}-\frac{1}{W_{n}} \sum_{i=1}^{n} w_{i} x_{i}}{\theta_{1}-\eta_{1}} \zeta\left(\theta_{1}\right) & +\frac{\frac{1}{W_{n}} \sum_{i=1}^{n} w_{i} x_{i}-\eta_{1}}{\theta_{1}-\eta_{1}} \zeta\left(\eta_{1}\right) \\
& \leq \zeta\left(\eta_{1}\right)+\zeta\left(\theta_{1}\right)-\frac{1}{W_{n}} \sum_{i=1}^{n} w_{i} \zeta\left(x_{i}\right) .
\end{aligned}
$$

Proof. Since $\zeta$ is a continuous and convex function, the same is also true for the function $\psi:\left[\eta_{1}, \theta_{1}\right] \subseteq I \rightarrow \mathbb{R}$ defined by $\psi(t)=\zeta\left(\eta_{1}+\theta_{1}-t\right)$, defined on $\left[\eta_{1}, \theta_{1}\right] \subseteq$ $I \subset \mathbb{R}$. By Jensen's Inequality, we have

$$
\psi\left(\frac{1}{W_{n}} \sum_{i=1}^{n} w_{i} x_{i}\right) \leq \frac{1}{W_{n}} \sum_{i=1}^{n} w_{i} \psi\left(x_{i}\right),
$$

which is turn gives us following inequality by using $\zeta$,

$$
\begin{aligned}
& \zeta\left(\eta_{1}+\theta_{1}-\frac{1}{W_{n}} \sum_{i=1}^{n} w_{i} x_{i}\right) \leq \frac{1}{W_{n}} \sum_{i=1}^{n} w_{i} \zeta\left(\eta_{1}+\theta_{1}-x_{i}\right) \\
& \leq \frac{\theta_{1}-\frac{1}{W_{n}} \sum_{i=1}^{n} w_{i} x_{i}}{\theta_{1}-\eta_{1}} \psi\left(\eta_{1}\right)+\frac{\frac{1}{W_{n}} \sum_{i=1}^{n} w_{i} x_{i}-\eta_{1}}{\theta_{1}-\eta_{1}} \psi\left(\theta_{1}\right) \quad(\text { by } \quad \text { Lemma } \\
& =\frac{\theta_{1}-\frac{1}{W_{n}} \sum_{i=1}^{n} w_{i} x_{i}}{\theta_{1}-\eta_{1}} \zeta\left(\theta_{1}\right)+\frac{\frac{1}{W_{n}} \sum_{i=1}^{n} w_{i} x_{i}-\eta_{1}}{\theta_{1}-\eta_{1}} \zeta\left(\eta_{1}\right) \\
& =\zeta\left(\eta_{1}\right)+\zeta\left(\theta_{1}\right)-\left[\frac{\theta_{1}-\frac{1}{W_{n}} \sum_{i=1}^{n} w_{i} x_{i}}{\theta_{1}-\eta_{1}} \zeta\left(\eta_{1}\right)+\frac{\frac{1}{W_{n}} \sum_{i=1}^{n} w_{i} x_{i}-\eta_{1}}{\theta_{1}-\eta_{1}} \zeta\left(\theta_{1}\right)\right] \\
& \left.\leq \zeta\left(\eta_{1}\right)+\zeta\left(\theta_{1}\right)-\frac{1}{W_{n}} \sum_{i=1}^{n} w_{i} \zeta\left(x_{i}\right) . \quad \text { (by } \quad \text { Theorem } \quad 1.6\right)
\end{aligned}
$$

Theorem 4.4. Let $\zeta: I \longrightarrow \mathbb{R}$ be a convex function and $\left[\eta_{1}, \theta_{1}\right] \subseteq I, \mathbf{x}=$ $\left(x_{1}, \ldots, x_{n}\right) \in\left[\eta_{1}, \theta_{1}\right]^{n}$ and $\mathbf{w}=\left(w_{1}, \ldots, w_{n}\right)$ be a positive $n$-tuple such that $W_{n}=\sum_{i=1}^{n} w_{i}>0$, then

$$
\begin{aligned}
& \zeta\left(\eta_{1}+\theta_{1}-\frac{1}{W_{n}} \sum_{i=1}^{n} w_{i} x_{i}\right) \leq \frac{1}{W_{n}} \sum_{i=1}^{n} w_{i} \zeta\left(\eta_{1}+\theta_{1}-x_{i}\right) \\
& \leq \frac{\theta_{1}-\frac{1}{W_{n}} \sum_{i=1}^{n} w_{i} x_{i}}{\theta_{1}-\eta_{1}} \zeta\left(\theta_{1}\right)+\frac{\frac{1}{w_{n}} \sum_{i=1}^{n} w_{i} x_{i}-\eta_{1}}{\theta_{1}-\eta_{1}} \zeta\left(\eta_{1}\right) \\
& -\left[\frac{1}{W_{n}} \sum_{i=1}^{n} w_{i}\left(\frac{1}{2}-\frac{1}{\theta_{1}-\eta_{1}}\left|x_{i}-\frac{\eta_{1}+\theta_{1}}{2}\right|\right)\right] \delta_{\zeta}
\end{aligned}
$$




$$
\begin{aligned}
& \leq \zeta\left(\eta_{1}\right)+\zeta\left(\theta_{1}\right)-\frac{1}{W_{n}} \sum_{i=1}^{n} w_{i} \zeta\left(x_{i}\right)-\left[1-\frac{2}{\theta_{1}-\eta_{1}} \frac{1}{W_{n}} \sum_{i=1}^{n} w_{i}\left|x_{i}-\frac{\eta_{1}+\theta_{1}}{2}\right|\right] \delta_{\zeta} \\
& \leq \zeta\left(\eta_{1}\right)+\zeta\left(\theta_{1}\right)-\frac{1}{W_{n}} \sum_{i=1}^{n} w_{i} \zeta\left(x_{i}\right) .
\end{aligned}
$$

where $\delta_{\zeta}=\zeta\left(\eta_{1}\right)+\zeta\left(\theta_{1}\right)-2 \zeta\left(\frac{\eta_{1}+\theta_{1}}{2}\right)$.

Proof. Using first inequality of the series (4.2) and applying inequality (1.7) first to function $\eta_{1}+\theta_{1}-x_{i}$ and then to the function $x_{i}$, we obtain

$$
\begin{aligned}
& \zeta\left(\eta_{1}+\theta_{1}-\frac{1}{W_{n}} \sum_{i=1}^{n} w_{i} x_{i}\right) \\
& \leq \frac{1}{W_{n}} \sum_{i=1}^{n} w_{i} \zeta\left(\eta_{1}+\theta_{1}-x_{i}\right) \\
& \leq \frac{\theta_{1}-\frac{1}{W_{n}} \sum_{i=1}^{n} w_{i} x_{i}}{\theta_{1}-\eta_{1}} \zeta\left(\theta_{1}\right)+\frac{\frac{1}{W_{n}} \sum_{i=1}^{n} w_{i} x_{i}-\eta_{1}}{\theta_{1}-\eta_{1}} \zeta\left(\eta_{1}\right) \\
& -\left[\frac{1}{W_{n}} \sum_{i=1}^{n} w_{i}\left(\frac{1}{2}-\frac{1}{\theta_{1}-\eta_{1}}\left|x_{i}-\frac{\eta_{1}+\theta_{1}}{2}\right|\right)\right] \delta_{\zeta} \\
& =\zeta\left(\eta_{1}\right)+\zeta\left(\theta_{1}\right)-\left[\frac{\theta_{1}-\frac{1}{W_{n}} \sum_{i=1}^{n} w_{i} x_{i}}{\theta_{1}-\eta_{1}} \zeta\left(\eta_{1}\right)+\frac{\frac{1}{W_{n}} \sum_{i=1}^{n} w_{i} x_{i}-\eta_{1}}{\theta_{1}-\eta_{1}} \zeta\left(\theta_{1}\right)\right] \\
& -\frac{1}{W_{n}} \sum_{i=1}^{n} w_{i}\left(\frac{1}{2}-\frac{1}{\theta_{1}-\eta_{1}}\left|x_{i}-\frac{\eta_{1}+\theta_{1}}{2}\right|\right) \delta_{\zeta} \\
& \leq \zeta\left(\eta_{1}\right)+\zeta\left(\theta_{1}\right)-\frac{1}{W_{n}} \sum_{i=1}^{n} w_{i} \zeta\left(x_{i}\right)-2 \frac{1}{W_{n}} \sum_{i=1}^{n} w_{i}\left(\frac{1}{2}-\frac{1}{\theta_{1}-\eta_{1}}\left|x_{i}-\frac{\eta_{1}+\theta_{1}}{2}\right|\right) \delta_{\zeta} \\
& =\zeta\left(\eta_{1}\right)+\zeta\left(\theta_{1}\right)-\frac{1}{W_{n}} \sum_{i=1}^{n} w_{i} \zeta\left(x_{i}\right)-\left(1-\frac{2}{\theta_{1}-\eta_{1}} \frac{1}{W_{n}} \sum_{i=1}^{n} w_{i}\left(\left|x_{i}-\frac{\eta_{1}+\theta_{1}}{2}\right|\right) \delta_{\zeta}\right. \\
& \leq \zeta\left(\eta_{1}\right)+\zeta\left(\theta_{1}\right)-\frac{1}{W_{n}} \sum_{i=1}^{n} w_{i} \zeta\left(x_{i}\right) .
\end{aligned}
$$

This simple provable fact is the justification of the above mentioned last inequality. $\delta_{\zeta}=\zeta\left(\eta_{1}\right)+\zeta\left(\theta_{1}\right)-2 \zeta\left(\frac{\eta_{1}+\theta_{1}}{2}\right) \geq 0$ and $1-\frac{2}{\theta_{1}-\eta_{1}}\left(\left|f-\frac{\eta_{1}+\theta_{1}}{2}\right|\right) \geq 0$.

Now here we state and prove another result which provides generalized refinement of discrete type Jensen-Mercer inequality. 
Theorem 4.5. Under the assumptions of Theorem 4.4, we have

$$
\begin{aligned}
& \zeta\left(\eta_{1}+\theta_{1}-\frac{1}{W_{n}} \sum_{i=1}^{n} w_{i} x_{i}\right) \\
& \leq \frac{\theta_{1}-\frac{1}{W_{n}} \sum_{i=1}^{n} w_{i} x_{i}}{\theta_{1}-\eta_{1}} \zeta\left(\theta_{1}\right)+\frac{\frac{1}{W_{n}} \sum_{i=1}^{n} w_{i} x_{i}-\eta_{1}}{\theta_{1}-\eta_{1}} \zeta\left(\eta_{1}\right) \\
& -\left[\frac{1}{2}-\frac{1}{\theta_{1}-\eta_{1}}\left|\frac{1}{W_{n}} \sum_{i=1}^{n} w_{i} x_{i}-\frac{\eta_{1}+\theta_{1}}{2}\right|\right] \delta_{\zeta} \\
& \leq \zeta\left(\eta_{1}\right)+\zeta\left(\theta_{1}\right)-\frac{1}{W_{n}} \sum_{i=1}^{n} w_{i} \zeta\left(x_{i}\right) \\
& -\left[1-\frac{1}{\theta_{1}-\eta_{1}}\left(\frac{1}{W_{n}} \sum_{i=1}^{n} w_{i}\left|x_{i}-\frac{\eta_{1}+\theta_{1}}{2}\right|+\left|\frac{1}{W_{n}} \sum_{i=1}^{n} w_{i} x_{i}-\frac{\eta_{1}+\theta_{1}}{2}\right|\right)\right] \delta_{\zeta} \\
& \leq \zeta\left(\eta_{1}\right)+\zeta\left(\theta_{1}\right)-\frac{1}{W_{n}} \sum_{i=1}^{n} w_{i} \zeta\left(x_{i}\right)-\left[1-\frac{2}{\theta_{1}-\eta_{1}} \frac{1}{W_{n}} \sum_{i=1}^{n} w_{i}\left(\left|x_{i}-\frac{\eta_{1}+\theta_{1}}{2}\right|\right)\right] \delta_{\zeta} \\
& \leq \zeta\left(\eta_{1}\right)+\zeta\left(\theta_{1}\right)-\frac{1}{W_{n}} \sum_{i=1}^{n} w_{i} \zeta\left(x_{i}\right) .
\end{aligned}
$$

where $\delta_{\zeta}$ is as defined in Theorem 4.4.

Proof. Inequality (1.7) gives us

$$
\begin{aligned}
& \frac{1}{W_{n}} \sum_{i=1}^{n} w_{i} \zeta\left(x_{i}\right) \leq \frac{\theta_{1}-\frac{1}{W_{n}} \sum_{i=1}^{n} w_{i} x_{i}}{\theta_{1}-\eta_{1}} \zeta\left(\eta_{1}\right)+\frac{\frac{1}{W_{n}} \sum_{i=1}^{n} w_{i} x_{i}-\eta_{1}}{\theta_{1}-\eta_{1}} \zeta\left(\theta_{1}\right) \\
& -\frac{1}{W_{n}} \sum_{i=1}^{n} w_{i}\left[\frac{1}{2}-\frac{1}{\theta_{1}-\eta_{1}}\left|x_{i}-\frac{\eta_{1}+\theta_{1}}{2}\right|\right] \delta_{\zeta}
\end{aligned}
$$

Let the functions $p, q:\left[\eta_{1}, \theta_{1}\right] \rightarrow[0,1]$ be defined by, $p(t)=\frac{\theta_{1}-t}{\theta_{1}-\eta_{1}}, q(t)=\frac{t-\eta_{1}}{\theta_{1}-\eta_{1}}$ for any $t \in\left[\eta_{1}, \theta_{1}\right]$, we can write

$$
\zeta(t)=\zeta\left(\frac{\theta_{1}-t}{\theta_{1}-\eta_{1}} \eta_{1}+\frac{t-\eta_{1}}{\theta_{1}-\eta_{1}} \theta_{1}\right)=\zeta\left(p(t) \eta_{1}+q(t) \theta_{1}\right)
$$

By Lemma 1.11 it follows

$$
\zeta(t) \leq p(t) \zeta\left(\eta_{1}\right)+q(t) \zeta\left(\theta_{1}\right)-\min \{p(t), q(t)\} \delta_{\zeta}
$$

where $t=\left(\frac{\theta_{1}-t}{\theta_{1}-\eta_{1}} \eta_{1}+\frac{t-\eta_{1}}{\theta_{1}-\eta_{1}} \theta_{1}\right)$ and $\delta_{\zeta}=\zeta\left(\eta_{1}\right)+\zeta\left(\theta_{1}\right)-2 \zeta\left(\frac{\eta_{1}+\theta_{1}}{2}\right)$. It can be written in the form

$$
\zeta(t) \leq \frac{\theta_{1}-t}{\theta_{1}-\eta_{1}} \zeta\left(\eta_{1}\right)+\frac{t-\eta_{1}}{\theta_{1}-\eta_{1}} \zeta\left(\theta_{1}\right)-\left(\frac{1}{2}-\frac{1}{\theta_{1}-\eta_{1}}\left|t-\frac{\eta_{1}+\theta_{1}}{2}\right|\right) \delta_{\zeta}
$$


substituting $t=\frac{1}{W_{n}} \sum_{i=1}^{n} w_{i} x_{i}$, clearly $\frac{1}{W_{n}} \sum_{i=1}^{n} w_{i} x_{i} \in\left[\eta_{1}, \theta_{1}\right]$, we get

$$
\begin{array}{r}
\zeta\left(\frac{1}{W_{n}} \sum_{i=1}^{n} w_{i} x_{i}\right) \leq \frac{\theta_{1}-\frac{1}{W_{n}} \sum_{i=1}^{n} w_{i} x_{i}}{\theta_{1}-\eta_{1}} \zeta\left(\eta_{1}\right)+\frac{\frac{1}{W_{n}} \sum_{i=1}^{n} w_{i} x_{i}-\eta_{1}}{\theta_{1}-\eta_{1}} \zeta\left(\theta_{1}\right) \\
-\left(\frac{1}{2}-\frac{1}{\theta_{1}-\eta_{1}}\left|\frac{1}{W_{n}} \sum_{i=1}^{n} w_{i} x_{i}-\frac{\eta_{1}+\theta_{1}}{2}\right|\right) \delta_{\zeta} .
\end{array}
$$

Now applying above inequality (4.4) on $\eta_{1}+\theta_{1}-x_{i}$ and using inequality (4.3), we have

$$
\begin{aligned}
& \zeta\left(\eta_{1}+\theta_{1}-\frac{1}{W_{n}} \sum_{i=1}^{n} w_{i} x_{i}\right) \leq \frac{\theta_{1}-\frac{1}{W_{n}} \sum_{i=1}^{n} w_{i} x_{i}}{\theta_{1}-\eta_{1}} \zeta\left(\theta_{1}\right)+\frac{\frac{1}{W_{n}} \sum_{i=1}^{n} w_{i} x_{i}-\eta_{1}}{\theta_{1}-\eta_{1}} \zeta\left(\eta_{1}\right) \\
& -\left[\frac{1}{2}-\left(\frac{1}{\theta_{1}-\eta_{1}}\left|\frac{1}{W_{n}} \sum_{i=1}^{n} w_{i} x_{i}-\frac{\eta_{1}+\theta_{1}}{2}\right|\right)\right] \delta_{\zeta} \\
& =\zeta\left(\eta_{1}\right)+\zeta\left(\theta_{1}\right)-\left[\frac{\theta_{1}-\frac{1}{W_{n}} \sum_{i=1}^{n} w_{i} x_{i}}{\theta_{1}-\eta_{1}} \zeta\left(\eta_{1}\right)+\frac{\frac{1}{W_{n}} \sum_{i=1}^{n} w_{i} x_{i}-\eta_{1}}{\theta_{1}-\eta_{1}} \zeta\left(\theta_{1}\right)\right] \\
& -\left[\frac{1}{2}-\frac{1}{\theta_{1}-\eta_{1}}\left|\frac{1}{W_{n}} \sum_{i=1}^{n} w_{i} x_{i}-\frac{\eta_{1}+\theta_{1}}{2}\right|\right] \delta_{\zeta} \\
& \leq \zeta\left(\eta_{1}\right)+\zeta\left(\theta_{1}\right)-\frac{1}{W_{n}} \sum_{i=1}^{n} w_{i} \zeta\left(x_{i}\right)-\left[\frac{1}{W_{n}} \sum_{i=1}^{n} w_{i}\left(\frac{1}{2}-\frac{1}{\theta_{1}-\eta_{1}}\left|x_{i}-\frac{\eta_{1}+\theta_{1}}{2}\right|\right)\right] \delta_{\zeta} \\
& -\left[\frac{1}{2}-\frac{1}{\theta_{1}-\eta_{1}}\left|\frac{1}{W_{n}} \sum_{i=1}^{n} w_{i} x_{i}-\frac{\eta_{1}+\theta_{1}}{2}\right|\right] \delta_{\zeta} \\
& =\zeta\left(\eta_{1}\right)+\zeta\left(\theta_{1}\right)-\frac{1}{W_{n}} \sum_{i=1}^{n} w_{i} \zeta\left(x_{i}\right) \\
& -\left[1-\frac{1}{\theta_{1}-\eta_{1}}\left(\frac{1}{W_{n}} \sum_{i=1}^{n} w_{i}\left|x_{i}-\frac{\eta_{1}+\theta_{1}}{2}\right|\right)+\left|\frac{1}{W_{n}} \sum_{i=1}^{n} w_{i} x_{i}-\frac{\eta_{1}+\theta_{1}}{2}\right|\right] \delta_{\zeta} \\
& \leq \zeta\left(\eta_{1}\right)+\zeta\left(\theta_{1}\right)-\frac{1}{W_{n}} \sum_{i=1}^{n} w_{i} \zeta\left(x_{i}\right)-\left[1-\frac{2}{\theta_{1}-\eta_{1}} \frac{1}{W_{n}} \sum_{i=1}^{n} w_{i}\left|x_{i}-\frac{\eta_{1}+\theta_{1}}{2}\right|\right] \delta_{\zeta} .
\end{aligned}
$$

The last inequality is obtained by applying following inequality on the convex function $|x|$ so that

$$
\begin{aligned}
\left|\frac{1}{W_{n}} \sum_{i=1}^{n} w_{i} x_{i}-\frac{\eta_{1}+\theta_{1}}{2}\right| & =\left|\frac{1}{W_{n}} \sum_{i=1}^{n} w_{i}\left(x_{i}-\frac{\eta_{1}+\theta_{1}}{2}\right)\right| \\
& \leq \frac{1}{W_{n}} \sum_{i=1}^{n} w_{i}\left|x_{i}-\frac{\eta_{1}+\theta_{1}}{2}\right| .
\end{aligned}
$$

Using Theorem 4.5. we can obtain an upper bound for the Jensen's difference,

$$
\frac{1}{W_{n}} \sum_{i=1}^{n} w_{i} \zeta\left(x_{i}\right)-\zeta\left(\frac{1}{W_{n}} \sum_{i=1}^{n} w_{i} x_{i}\right) .
$$


Corollary 4.6. Under the assumptions of Theorem 4.4, we have

$$
\begin{aligned}
& \frac{1}{W_{n}} \sum_{i=1}^{n} w_{i} \zeta\left(x_{i}\right)-\zeta\left(\frac{1}{W_{n}} \sum_{i=1}^{n} w_{i} x_{i}\right) \\
& \leq \frac{1}{\theta_{1}-\eta_{1}}\left(\frac{1}{W_{n}} \sum_{i=1}^{n} w_{i}\left|x_{i}-\frac{\eta_{1}+\theta_{1}}{2}\right|+\left|\frac{1}{W_{n}} \sum_{i=1}^{n} w_{i} x_{i}-\frac{\eta_{1}+\theta_{1}}{2}\right|\right) \delta_{\zeta}
\end{aligned}
$$

where $\delta_{\zeta}$ is as defined in Theorem 4.4 .

Proof. Theorem 4.5 gives us

$$
\begin{aligned}
& \frac{1}{W_{n}} \sum_{i=1}^{n} w_{i} \zeta\left(x_{i}\right) \leq \zeta\left(\eta_{1}\right)+\zeta\left(\theta_{1}\right)-\zeta\left(\eta_{1}+\theta_{1}-\frac{1}{W_{n}} \sum_{i=1}^{n} w_{i} x_{i}\right) \\
& -\left[1-\frac{1}{\theta_{1}-\eta_{1}}\left(\frac{1}{W_{n}} \sum_{i=1}^{n} w_{i}\left|x_{i}-\frac{\eta_{1}+\theta_{1}}{2}\right|\right)+\left|\frac{1}{W_{n}} \sum_{i=1}^{n} w_{i} x_{i}-\frac{\eta_{1}+\theta_{1}}{2}\right|\right] \delta_{\zeta} .
\end{aligned}
$$

By adding $-\zeta\left(\frac{1}{W_{n}} \sum_{i=1}^{n} w_{i} x_{i}\right)$ on the both sides, we get

$$
\begin{aligned}
& \frac{1}{W_{n}} \sum_{i=1}^{n} w_{i} \zeta\left(x_{i}\right)-\zeta\left(\frac{1}{W_{n}} \sum_{i=1}^{n} w_{i} x_{i}\right) \\
& \leq \zeta\left(\eta_{1}\right)+\zeta\left(\theta_{1}\right)-\zeta\left(\eta_{1}+\theta_{1}-\frac{1}{W_{n}} \sum_{i=1}^{n} w_{i} x_{i}\right)-\zeta\left(\frac{1}{W_{n}} \sum_{i=1}^{n} w_{i} x_{i}\right) \\
& -\left[1-\frac{1}{\theta_{1}-\eta_{1}}\left(\frac{1}{W_{n}} \sum_{i=1}^{n} w_{i}\left|x_{i}-\frac{\eta_{1}+\theta_{1}}{2}\right|+\left|\frac{1}{W_{n}} \sum_{i=1}^{n} w_{i} x_{i}-\frac{\eta_{1}+\theta_{1}}{2}\right|\right)\right] \delta_{\zeta} \\
& =\zeta\left(\eta_{1}\right)+\zeta\left(\theta_{1}\right)-\left[\zeta\left(\eta_{1}+\theta_{1}-\frac{1}{W_{n}} \sum_{i=1}^{n} w_{i} x_{i}\right)+\zeta\left(\frac{1}{W_{n}} \sum_{i=1}^{n} w_{i} x_{i}\right)\right] \\
& -\left[1-\frac{1}{\theta_{1}-\eta_{1}}\left(\frac{1}{W_{n}} \sum_{i=1}^{n} w_{i}\left|x_{i}-\frac{\eta_{1}+\theta_{1}}{2}\right|+\left|\frac{1}{W_{n}} \sum_{i=1}^{n} w_{i} x_{i}-\frac{\eta_{1}+\theta_{1}}{2}\right|\right)\right] \delta_{\zeta} .
\end{aligned}
$$

Since $\zeta$ is convex function it follows,

$$
\zeta\left(\eta_{1}+\theta_{1}-\frac{1}{W_{n}} \sum_{i=1}^{n} w_{i} x_{i}\right)+\zeta\left(\frac{1}{W_{n}} \sum_{i=1}^{n} w_{i} x_{i}\right) \geq 2 \zeta\left(\frac{\eta_{1}+\theta_{1}}{2}\right)
$$


Hence, finally we have

$$
\begin{aligned}
& \frac{1}{W_{n}} \sum_{i=1}^{n} w_{i} \zeta\left(x_{i}\right)-\zeta\left(\frac{1}{W_{n}} \sum_{i=1}^{n} w_{i} x_{i}\right) \\
& \leq \zeta\left(\eta_{1}\right)+\zeta\left(\theta_{1}\right)-2 \zeta\left(\frac{\eta_{1}+\theta_{1}}{2}\right) \\
& -\left[1-\frac{1}{\theta_{1}-\eta_{1}}\left(\frac{1}{W_{n}} \sum_{i=1}^{n} w_{i}\left|x_{i}-\frac{\eta_{1}+\theta_{1}}{2}\right|+\left|\frac{1}{W_{n}} \sum_{i=1}^{n} w_{i} x_{i}-\frac{\eta_{1}+\theta_{1}}{2}\right|\right)\right] \delta_{\zeta} \\
& =\frac{1}{\theta_{1}-\eta_{1}}\left[\frac{1}{W_{n}} \sum_{i=1}^{n} w_{i}\left|x_{i}-\frac{\eta_{1}+\theta_{1}}{2}\right|+\left|\frac{1}{W_{n}} \sum_{i=1}^{n} w_{i} x_{i}-\frac{\eta_{1}+\theta_{1}}{2}\right|\right] \delta_{\zeta} .
\end{aligned}
$$

\section{Refinements Of Integral Jensen-Mercer Inequality}

Theorem 5.1. Let $(\Omega, P, \mu)$ be a probability measure space and $f: \Omega \longrightarrow\left[\eta_{1}, \theta_{1}\right]$ is a measurable and integrable function. Then for any continuous convex function $\zeta:\left[\eta_{1}, \theta_{1}\right] \longrightarrow \mathbb{R}$, we have

$$
\int_{\Omega} \zeta(f) d \mu \leq \frac{\theta_{1}-\int_{\Omega} f d \mu}{\theta_{1}-\eta_{1}} \zeta\left(\eta_{1}\right)+\frac{\int_{\Omega} f d \mu-\eta_{1}}{\theta_{1}-\eta_{1}} \zeta\left(\theta_{1}\right)-\left(\int_{\Omega} \check{f} d \mu\right) \delta_{\zeta}
$$

where $\check{f}=\frac{1}{2}-\frac{1}{\theta_{1}-\eta_{1}}\left|f-\frac{\eta_{1}+\theta_{1}}{2}\right|, \delta_{\zeta}=\zeta\left(\eta_{1}\right)+\zeta\left(\theta_{1}\right)-2 \zeta\left(\frac{\eta_{1}+\theta_{1}}{2}\right)$.

Proof. We have, $\eta_{1} \leq \int_{\Omega} f d \mu \leq \theta_{1}$.

Let the functions $p, q:\left[\eta_{1}, \theta_{1}\right] \longrightarrow \mathbb{R}$ be defined by, $p(x)=\frac{\theta_{1}-x}{\theta_{1}-\eta_{1}}, \quad q(x)=\frac{x-\eta_{1}}{\theta_{1}-\eta_{1}} \quad$ for any $x \in\left[\eta_{1}, \theta_{1}\right]$, we can write

$$
\zeta(x)=\zeta\left(\frac{\theta_{1}-x}{\theta_{1}-\eta_{1}} \eta_{1}+\frac{x-\eta_{1}}{\theta_{1}-\eta_{1}} \theta_{1}\right)=\zeta\left(p(x) \eta_{1}+q(x) \theta_{1}\right)
$$

By Lemma 1.11 it follows that

$$
\zeta(x) \leq p(x) \zeta\left(\eta_{1}\right)+q(x) \zeta\left(\theta_{1}\right)-\min \{p(x), q(x)\} \delta_{\zeta}
$$

now integrating the above inequality with respect to $\mu$ and by putting $x=f$, we obtain

$$
\int_{\Omega} \zeta(f) d \mu \leq \zeta\left(\eta_{1}\right) \int_{\Omega} p(f) d \mu+\zeta\left(\theta_{1}\right) \int_{\Omega} q(f) d \mu-\left(\int_{\Omega} \check{f} d \mu\right) \delta_{\zeta} .
$$

where the function $\check{f}$ is defined as,

$$
\begin{aligned}
& \check{f}(x)=\min \{p(f), q(f)\}(x)=\frac{1}{2}-\frac{\left|f-\frac{\eta_{1}+\theta_{1}}{2}\right|}{\theta_{1}-\eta_{1}} . \\
& \text { since } p \text { and } q \text { are integrable functions so we have } \\
& \int_{\Omega} p(f) d \mu=p\left(\int_{\Omega} f d \mu\right) \text { and } \int_{\Omega} q(f) d \mu=q\left(\int_{\Omega} f d \mu\right) .
\end{aligned}
$$

Hence,

$$
\int_{\Omega} \zeta(f) d \mu \leq p\left(\int_{\Omega} f d \mu\right) \zeta\left(\eta_{1}\right)+q\left(\int_{\Omega} f d \mu\right) \zeta\left(\theta_{1}\right)-\left(\int_{\Omega} \check{f} d \mu\right) \delta_{\zeta} .
$$

which is infact (5.1). 
Theorem 5.2. Under the assumptions of Theorem 5.1 the following inequality holds

$$
\begin{aligned}
& \zeta\left(\eta_{1}+\theta_{1}-\int_{\Omega} f d \mu\right) \leq \int_{\Omega} \zeta\left(\eta_{1}+\theta_{1}-f\right) d \mu \\
& \leq \frac{\theta_{1}-\int_{\Omega} f d \mu}{\theta_{1}-\eta_{1}} \zeta\left(\theta_{1}\right)+\frac{\int_{\Omega} f d \mu-\eta_{1}}{\theta_{1}-\eta_{1}} \zeta\left(\eta_{1}\right) \\
& -\left[\int_{\Omega}\left(\frac{1}{2}-\frac{1}{\theta_{1}-\eta_{1}}\left|f-\frac{\eta_{1}+\theta_{1}}{2}\right|\right) d \mu\right] \delta_{\zeta} \\
& \leq \zeta\left(\eta_{1}\right)+\zeta\left(\theta_{1}\right)-\int_{\Omega} \zeta(f) d \mu-\left[1-\frac{2}{\theta_{1}-\eta_{1}} \int_{\Omega}\left|f-\frac{\eta_{1}+\theta_{1}}{2}\right| d \mu\right] \delta_{\zeta} \\
& \leq \zeta\left(\eta_{1}\right)+\zeta\left(\theta_{1}\right)-\int_{\Omega} \zeta(f) d \mu .
\end{aligned}
$$

where, $\delta_{\zeta}=\zeta\left(\eta_{1}\right)+\zeta\left(\theta_{1}\right)-2 \zeta\left(\frac{\eta_{1}+\theta_{1}}{2}\right)$.

Proof. Using the first inequality from the series (1.13) and applying inequality (5.1) first to the function $\eta_{1}+\theta_{1}-f$ and then to the function $f$, we obtain

$$
\begin{aligned}
& \zeta\left(\eta_{1}+\theta_{1}-\int_{\Omega} f d \mu\right) \leq \int_{\Omega} \zeta\left(\eta_{1}+\theta_{1}-f\right) d \mu \\
& \leq \frac{\theta_{1}-\int_{\Omega} f d \mu}{\theta_{1}-\eta_{1}} \zeta\left(\theta_{1}\right)+\frac{\int_{\Omega} f d \mu-\eta_{1}}{\theta_{1}-\eta_{1}} \zeta\left(\eta_{1}\right) \\
& -\left[\int_{\Omega}\left(\frac{1}{2}-\frac{1}{\theta_{1}-\eta_{1}}\left|f-\frac{\eta_{1}+\theta_{1}}{2}\right|\right) d \mu\right] \delta_{\zeta} \\
& \leq \zeta\left(\eta_{1}\right)+\zeta\left(\theta_{1}\right)-\left[\frac{\theta_{1}-\int_{\Omega} f d \mu}{\theta_{1}-\eta_{1}} \zeta\left(\eta_{1}\right)+\frac{\int_{\Omega} f d \mu-\eta_{1}}{\theta_{1}-\eta_{1}} \zeta\left(\theta_{1}\right)\right] \\
& -\left[\int_{\Omega}\left(\frac{1}{2}-\frac{1}{\theta_{1}-\eta_{1}}\left|f-\frac{\eta_{1}+\theta_{1}}{2}\right|\right) d \mu\right] \delta_{\zeta} \\
& \leq \zeta\left(\eta_{1}\right)+\zeta\left(\theta_{1}\right)-\int_{\Omega} \zeta(f) d \mu-2\left[\int_{\Omega}\left(\frac{1}{2}-\frac{1}{\theta_{1}-\eta_{1}}\left|f-\frac{\eta_{1}+\theta_{1}}{2}\right|\right) d \mu\right] \delta_{\zeta} \\
& \leq \zeta\left(\eta_{1}\right)+\zeta\left(\theta_{1}\right)-\int_{\Omega} \zeta(f) d \mu-\left[1-\frac{2}{\theta_{1}-\eta_{1}} \int_{\Omega}\left|f-\frac{\eta_{1}+\theta_{1}}{2}\right| d \mu\right] \delta_{\zeta} \\
& \leq \zeta\left(\eta_{1}\right)+\zeta\left(\theta_{1}\right)-\int_{\Omega} \zeta(f) d \mu .
\end{aligned}
$$

This simple provable fact is the justification of the above mentioned last inequality. $\delta_{\zeta}=\zeta\left(\eta_{1}\right)+\zeta\left(\theta_{1}\right)-2 \zeta\left(\frac{\eta_{1}+\theta_{1}}{2}\right) \geq 0$ and $\left(1-\frac{2}{\theta_{1}-\eta_{1}}\left|f-\frac{\eta_{1}+\theta_{1}}{2}\right|\right) \geq 0$. 
Theorem 5.3. Under the same assumptions of Theorem 5.1 the following inequality holds

$$
\begin{aligned}
& \zeta\left(\eta_{1}+\theta_{1}-\int_{\Omega} f d \mu\right) \leq \frac{\theta_{1}-\int_{\Omega} f d \mu}{\theta_{1}-\eta_{1}} \zeta\left(\theta_{1}\right)+\frac{\int_{\Omega} f d \mu-\eta_{1}}{\theta_{1}-\eta_{1}} \zeta\left(\eta_{1}\right) \\
& -\left(\frac{1}{2}-\frac{1}{\theta_{1}-\eta_{1}}\left|\int_{\Omega} f d \mu-\frac{\eta_{1}+\theta_{1}}{2}\right|\right) \delta_{\zeta} \\
& \leq \zeta\left(\eta_{1}\right)+\zeta\left(\theta_{1}\right)-\int_{\Omega} \zeta(f) d \mu \\
& -\left[1-\frac{1}{\theta_{1}-\eta_{1}}\left(\int_{\Omega}\left|f-\frac{\eta_{1}+\theta_{1}}{2}\right| d \mu+\left|\int_{\Omega} f d \mu-\frac{\eta_{1}+\theta_{1}}{2}\right|\right)\right] \delta_{\zeta} \\
& \leq \zeta\left(\eta_{1}\right)+\zeta\left(\theta_{1}\right)-\int_{\Omega} \zeta(f) d \mu-\left[1-\frac{2}{\theta_{1}-\eta_{1}} \int_{\Omega}\left|f-\frac{\eta_{1}+\theta_{1}}{2}\right| d \mu\right] \delta_{\zeta} \\
& \leq \zeta\left(\eta_{1}\right)+\zeta\left(\theta_{1}\right)-\int_{\Omega} \zeta(f) d \mu .
\end{aligned}
$$

where $\delta_{\zeta}=\zeta\left(\eta_{1}\right)+\zeta\left(\theta_{1}\right)-2 \zeta\left(\frac{\eta_{1}+\theta_{1}}{2}\right)$.

Proof. Theorem 5.1 for the function $f$ gives us

$$
\begin{aligned}
& \int_{\Omega} \zeta(f) d \mu \leq \frac{\theta_{1}-\int_{\Omega} f d \mu}{\theta_{1}-\eta_{1}} \zeta\left(\eta_{1}\right)+\frac{\int_{\Omega} f d \mu-\theta_{1}}{\theta_{1}-\eta_{1}} \zeta\left(\theta_{1}\right) \\
& -\left[\int_{\Omega}\left(\frac{1}{2}-\frac{1}{\theta_{1}-\eta_{1}}\left|f-\frac{\eta_{1}+\theta_{1}}{2}\right|\right) d \mu\right] \delta_{\zeta} .
\end{aligned}
$$

Let the functions $p, q:\left[\eta_{1}, \theta_{1}\right] \rightarrow[0,1]$ be defined by

$$
\begin{array}{r}
p(t)=\frac{\theta_{1}-t}{\theta_{1}-\eta_{1}}, \quad q(t)=\frac{t-\eta_{1}}{\theta_{1}-\eta_{1}} \quad \text { for any } \mathrm{t} \in\left[\eta_{1}, \theta_{1}\right], \text { we can write } \\
\zeta(t)=\zeta\left(\frac{\theta_{1}-t}{\theta_{1}-\eta_{1}} \eta_{1}+\frac{t-\eta_{1}}{\theta_{1}-\eta_{1}} \theta_{1}\right)=\zeta\left(p(t) \eta_{1}+q(t) \theta_{1}\right) .
\end{array}
$$

By Lemma 1.11 it follows that

$$
\begin{aligned}
\zeta(t) & \leq p(t) \zeta\left(\eta_{1}\right)+q(t) \zeta\left(\theta_{1}\right)-\min \{p(t), q(t)\} \delta_{\zeta} \\
\zeta(t) & \leq \frac{\theta_{1}-t}{\theta_{1}-\eta_{1}} \zeta\left(\eta_{1}\right)+\frac{t-\eta_{1}}{\theta_{1}-\eta_{1}} \zeta\left(\theta_{1}\right)-\left(\frac{1}{2}-\frac{1}{\theta_{1}-\eta_{1}}\left|t-\frac{\eta_{1}+\theta_{1}}{2}\right|\right) \delta_{\zeta} .
\end{aligned}
$$

substituting $t=\int_{\Omega} f d \mu$ we get

$$
\begin{aligned}
& \zeta\left(\int_{\Omega} f d \mu\right) \leq \frac{\theta_{1}-\int_{\Omega} f d \mu}{\theta_{1}-\eta_{1}} \zeta\left(\eta_{1}\right)+\frac{\int_{\Omega} f d \mu-\eta_{1}}{\theta_{1}-\eta_{1}} \zeta\left(\theta_{1}\right) \\
& -\left(\frac{1}{2}-\frac{1}{\theta_{1}-\eta_{1}}\left|\int_{\Omega} f d \mu-\frac{\eta_{1}+\theta_{1}}{2}\right|\right) \delta_{\zeta}
\end{aligned}
$$


now applying above inequality on $\eta_{1}+\theta_{1}-f$ and using (5.1) we obtain

$$
\begin{aligned}
\zeta & \left(\eta_{1}+\theta_{1}-\int_{\Omega} f d \mu\right) \leq \frac{\theta_{1}-\int_{\Omega} f d \mu}{\theta_{1}-\eta_{1}} \zeta\left(\theta_{1}\right)+\frac{\int_{\Omega} f d \mu-\eta_{1}}{\theta_{1}-\eta_{1}} \zeta\left(\eta_{1}\right) \\
- & \left(\frac{1}{2}-\frac{1}{\theta_{1}-\eta_{1}}\left|\int_{\Omega} f d \mu-\frac{\eta_{1}+\theta_{1}}{2}\right|\right) \delta_{\zeta} \\
= & \zeta\left(\eta_{1}\right)+\zeta\left(\theta_{1}\right)-\left[\frac{\theta_{1}-\int_{\Omega} f d \mu}{\theta_{1}-\eta_{1}} \zeta\left(\eta_{1}\right)+\frac{\int_{\Omega} f d \mu-\eta_{1}}{\theta_{1}-\eta_{1}} \zeta\left(\theta_{1}\right)\right] \\
- & {\left[\frac{1}{2}-\left(\frac{1}{\theta_{1}-\eta_{1}}\left|\int_{\Omega} f d \mu-\frac{\eta_{1}+\theta_{1}}{2}\right|\right)\right] \delta_{\zeta} } \\
\leq & \zeta\left(\eta_{1}\right)+\zeta\left(\theta_{1}\right)-\int_{\Omega} \zeta(f) d \mu-\left[\int_{\Omega}\left(\frac{1}{2}-\frac{1}{\theta_{1}-\eta_{1}}\left|f-\frac{\eta_{1}+\theta_{1}}{2}\right|\right) d \mu\right] \delta_{\zeta} \\
- & {\left[\frac{1}{2}-\frac{1}{\theta_{1}-\eta_{1}}\left|\int_{\Omega} f d \mu-\frac{\eta_{1}+\theta_{1}}{2}\right|\right] \delta_{\zeta} } \\
= & \zeta\left(\eta_{1}\right)+\zeta\left(\theta_{1}\right)-\int_{\Omega} \zeta(f) d \mu \\
- & {\left[1-\frac{1}{\theta_{1}-\eta_{1}} \int_{\Omega}\left|f-\frac{\eta_{1}+\theta_{1}}{2}\right| d \mu+\left|\int_{\Omega}(f) d \mu-\frac{\eta_{1}+\theta_{1}}{2}\right|\right] \delta_{\zeta} } \\
\leq & \zeta\left(\eta_{1}\right)+\zeta\left(\theta_{1}\right)-\int_{\Omega}(\zeta(f)) d \mu-\left[1-\frac{2}{\theta_{1}-\eta_{1}} \int_{\Omega}\left|f-\frac{\eta_{1}+\theta_{1}}{2}\right| d \mu\right] \delta_{\zeta} .
\end{aligned}
$$

The last inequality is obtained by applying inequality to the continuous and convex function $|x|$, so that

$$
\left|\int_{\Omega} f d \mu-\frac{\eta_{1}+\theta_{1}}{2}\right|=\left|\int_{\Omega}\left(f-\frac{\eta_{1}+\theta_{1}}{2}\right) d \mu\right| \leq \int_{\Omega}\left|f-\frac{\eta_{1}+\theta_{1}}{2}\right| d \mu .
$$

Using Theorem 5.3 we can get an upper bound for the difference $\int_{\Omega} \zeta(f) d \mu-\zeta\left(\int_{\Omega} f d \mu\right)$.

Corollary 5.4. Under the assumptions of Theorem 5.1 the following inequality holds

$$
\begin{aligned}
& \int_{\Omega} \zeta(f) d \mu-\zeta\left(\int_{\Omega} f d \mu\right) \\
& \leq \frac{1}{\theta_{1}-\eta_{1}}\left(\int_{\Omega}\left|f-\frac{\eta_{1}+\theta_{1}}{2}\right| d \mu+\left|\int_{\Omega} f d \mu-\frac{\eta_{1}+\theta_{1}}{2}\right|\right) \delta_{\zeta} .
\end{aligned}
$$

Proof. Since the proof is similar to the proof of Corollary 4.6, so we omit the details.

Remark 5.5. Similar results as given in section 4 and 5 can be obtained from [27].

\section{Applichtions}

In this section, we give applications in information theory, and generalized means obtained in Section 3 and 4. In this way our main results gives new bounds for Shannon entropy and generalize the relation among Arithmetic, Geometric, Harmonic and Power means. For the various properties of these means and relations 
among them we refer the reader to [5], [6] and [31].

6.1. Inequalities for Shannon's Entropy. Shannon entropy also known as measure of uncertainty, plays a vital role in information theory. It is also frequently applied in fields like population genetics, molecular ecology and dynamical system (see [26]). The entropy of a random variable is characterized regarding its probability distribution and can be shown to a decent measure of randomness or uncertainty. Shannon entropy as a basic concept in information theory measuring the average missing information in a random source. Shannon entropy permits to evaluate the normal least number of bits expected to encode a series of images dependent on the letters in order size and recurrence of symbols. Some recent bounds for Shannon entropy can be found in [12]. We extracted the following definition from [35].

Definition 6.1. Let $X$ be a random variable. If the probability distribution is given by

$$
P(X=i)=w_{i}, \quad w_{i}>0, i \in\{1, \ldots, n\} ; \text { and } \sum_{i=1}^{n} w_{i}=1
$$

then

$$
H(X)=\sum_{i=1}^{n} w_{i} \ln \frac{1}{w_{i}}
$$

For an application of some of our results in information theory, we shall give new bounds for Shannon's entropy H(X).

Theorem 6.2. Let $X$ be defined as above. Under the assumptions of Corollary 2.6, we have

$$
\ln n-H(X) \geq w_{i} \ln \left(\frac{2 w_{i}}{w_{i}+w_{j}}\right)+w_{j} \ln \left(\frac{2 w_{j}}{w_{i}+w_{j}}\right)
$$

Proof. It follows from Corollary 2.6 for the choice $\zeta(x)=-\ln x, x_{i}=\frac{1}{w_{i}}$ for each $i \in\{1, \ldots, n\}$ and by using elementary calculations.

6.2. Generalizing the relations among means. Let $J$ be a finite nonempty subsets of positive integers and let $\zeta: I \rightarrow \mathbb{R}$ be a function and $\left[\eta_{1}, \theta_{1}\right] \subseteq I$, $0<\eta_{1}<\theta_{1}$. Let $x=\left\{x_{i}\right\}_{i \in J}$ be a real monotonic sequence such that $x_{i} \in\left[\eta_{1}, \theta_{1}\right]$ for all $i \in J$ and $w=\left\{w_{i}\right\}_{i \in J}$ be a real sequence such that $w_{i} \neq 0$ for $i \in J$, and $\frac{1}{W_{J}} \sum_{i \in J} w_{i} x_{i} \in\left[\eta_{1}, \theta_{1}\right]$, where $W_{J}=\sum_{i \in J} w_{i}$. If we define

Generalized Arithmetic mean

$$
\widetilde{A}_{J}=\eta_{1}+\theta_{1}-\frac{1}{W_{J}} \sum_{i \in J} w_{i} x_{i}
$$

Generalized Geometric mean

$$
\widetilde{G}_{J}=\frac{\eta_{1} \theta_{1}}{\left(\prod_{i \in J} x_{i}^{w_{i}}\right)^{\frac{1}{W_{J}}}}
$$


Generalized Harmonic mean

$$
\widetilde{H}_{J}=\left(\eta_{1}^{-1}+\theta_{1}^{-1}-\frac{1}{W_{J}} \sum_{i \in J} w_{i} x_{i}^{-1}\right)^{-1}
$$

Generalized Power mean

$$
\widetilde{M}_{J}^{[r]}=\left(\eta_{1}^{r}+\theta_{1}^{r}-\frac{1}{W_{J}} \sum_{i \in J} w_{i} x_{i}^{r}\right)^{r}
$$

then we have the following relations among these means.

Remark 6.3. It is easy to see that

(1) (6.3) is direct consequence of (6.6) for $r=1$.

(2) (6.4) is direct consequence of (6.6) for $r=0$.

(3) (6.5) is direct consequence of (6.6) for $r=-1$.

Now, we define the relation between arithmetic and geometric means.

Theorem 6.4. Let $\zeta: I \rightarrow \mathbb{R}$ be a function and $\left[\eta_{1}, \theta_{1}\right] \subseteq I, 0<\eta_{1}<\theta_{1}$. Let $J$ and $K$ be finite non-empty subsets of positive integers such that $J \cap K=\phi$ and $J \cup K=\{1, \ldots, n\}$. Let $x=\left\{x_{i}\right\}_{i \in J \cup K}$ be a real monotonic sequence such that $x_{i} \in\left[\eta_{1}, \theta_{1}\right]$ and $w=\left\{w_{i}\right\}_{i \in J \cup K}$ be a real sequence such that $w_{i} \neq 0$ for $i \in J \cup K$. Further suppose $\frac{1}{W_{S}} \sum_{i \in S} w_{i} x_{i} \in\left[\eta_{1}, \theta_{1}\right] \quad(S=J, K, J \cup K)$ and $0<W_{S}<W_{J \cup K}$, where equality holds for $S=J \cup K$. If $\zeta$ is convex on $I$, then we have

$$
\begin{aligned}
& (i)\left(\frac{\widetilde{A}_{J \cup K}}{\widetilde{G}_{J \cup K}}\right)^{W_{J \cup K}} \geq\left(\frac{\widetilde{A}_{J}}{\widetilde{G}_{J}}\right)^{W_{J}}\left(\frac{\widetilde{A}_{K}}{\widetilde{G}_{K}}\right)^{W_{K}}, \\
& (i i) W_{J \cup K}\left(\widetilde{A}_{J \cup K}-\widetilde{G}_{J \cup K}\right) \geq W_{J}\left(\widetilde{A}_{J}-\widetilde{G}_{J}\right)+W_{K}\left(\widetilde{A}_{K}-\widetilde{G}_{K}\right) .
\end{aligned}
$$

Proof. (i) Applying the convex function $\zeta(x)=-\ln x$ for $x \in\left[\eta_{1}, \theta_{1}\right]$, to the functional $\widetilde{F}$ defined on index set $J \cup K$ as in (3.1).

$$
\begin{aligned}
& \widetilde{F}(J \cup K)=W_{J \cup K}\left[-\ln \eta_{1}-\ln \theta_{1}+\frac{1}{W_{J \cup K}} \sum_{i \in J \cup K} w_{i} \ln x_{i}+\right. \\
& \left.\ln \left(\eta_{1}+\theta_{1}-\frac{1}{W_{J \cup K}} \sum_{i \in J \cup K} w_{i} x_{i}\right)\right] .
\end{aligned}
$$

Using the property of $\ln$ in equation (6.9) and on further simplification we obtain

$$
\begin{aligned}
& \tilde{F}(J \cup K) \\
& =W_{J \cup K}\left[-\ln \frac{\eta_{1} \theta_{1}}{\left(\prod_{i \in J \cup K} x_{i}^{w_{i}}\right)^{\frac{1}{W_{J \cup K}}}}+\ln \left(\eta_{1}+\theta_{1}-\frac{1}{W_{J \cup K}} \sum_{i \in J \cup K} w_{i} x_{i}\right)\right] .
\end{aligned}
$$

Using (6.3) and (6.4) in equation (6.10) we have

$$
\widetilde{F}(J \cup K)=W_{J \cup K}\left[-\ln \widetilde{G}_{J \cup K}+\ln \widetilde{A}_{J \cup K}\right] .
$$


Using the property of ln we have

$$
\widetilde{F}(J \cup K)=W_{J \cup K} \ln \frac{\widetilde{A}_{J \cup K}}{\widetilde{G}_{J \cup K}}=\ln \left(\frac{\widetilde{A}_{J \cup K}}{\widetilde{G}_{J \cup K}}\right)^{W_{J \cup K}} .
$$

Similarly, applying the convex function $\zeta(x)=-\ln x$ for $x \in\left[\eta_{1}, \theta_{1}\right]$, to the functional $\widetilde{F}$ defined on index sets $J$ and $K$ as in (3.1), and then after simplification we get

$$
\begin{aligned}
& \widetilde{F}(J)=\ln \left(\frac{\widetilde{A}_{J}}{\widetilde{G}_{J}}\right)^{W_{J}}, \\
& \widetilde{F}(K)=\ln \left(\frac{\widetilde{A}_{K}}{\widetilde{G}_{K}}\right)^{W_{K}} .
\end{aligned}
$$

As all the assumptions of Theorem 3.1 are satisfied, hence by substituting values of $(6.11),(6.12)$ and $(6.13)$ in $(3.2)$ we get

$$
\ln \left(\frac{\widetilde{A}_{J \cup K}}{\widetilde{G}_{J \cup K}}\right)^{W_{J \cup K}} \geq \ln \left(\frac{\widetilde{A}_{J}}{\widetilde{G}_{J}}\right)^{W_{J}}+\ln \left(\frac{\widetilde{A}_{K}}{\widetilde{G}_{K}}\right)^{W_{K}} .
$$

Again using property of $\ln$ we get

$$
\ln \left(\frac{\widetilde{A}_{J \cup K}}{\widetilde{G}_{J \cup K}}\right)^{W_{J \cup K}} \geq \ln \left(\frac{\widetilde{A}_{J}}{\widetilde{G}_{J}}\right)^{W_{J}}\left(\frac{\widetilde{A}_{K}}{\widetilde{G}_{K}}\right)^{W_{K}} .
$$

Since exponential function is an increasing function, by applying it we will get our required result.

(ii) Applying the convex function $\zeta(x)=\exp x$ for $x \in\left[\eta_{1}, \theta_{1}\right]$, to the functional $\widetilde{F}$ defined on index set $J \cup K$ as in (3.1).

$$
\begin{aligned}
& \tilde{F}(J \cup K) \\
& =W_{J \cup K}\left[\exp \eta_{1}+\exp \theta_{1}-\frac{\sum_{i \in J \cup K} w_{i} \exp x_{i}}{W_{J \cup K}}-\exp \left(\eta_{1}+\theta_{1}-\frac{\sum_{i \in J \cup K} w_{i} x_{i}}{W_{J \cup K}}\right)\right],
\end{aligned}
$$

replacing $\eta_{1}, \theta_{1}$ and $x_{i}$ with $\ln \eta_{1}, \ln \theta_{1}$ and $\ln x_{i}$ in equation (6.14) and then after simplification we get

$$
\begin{aligned}
& \tilde{F}(J \cup K) \\
& =W_{J \cup K}\left[\eta_{1}+\theta_{1}-\frac{1}{W_{J \cup K}} \sum_{i \in J \cup K} w_{i} x_{i}-\frac{\eta_{1} \theta_{1}}{\left(\prod_{i \in J \cup K} x_{i}^{w_{i}}\right)^{\frac{1}{W_{J \cup K}}}}\right] .
\end{aligned}
$$

Using (6.3) and (6.4) in equation (6.15) we get

$$
\widetilde{F}(J \cup K)=W_{J \cup K}\left(\widetilde{A}_{J \cup K}-\widetilde{G}_{J \cup K}\right) .
$$

Similarly, applying the convex function $\zeta(x)=\exp x$ for $x \in\left[\eta_{1}, \theta_{1}\right]$, to the functional $\widetilde{F}$ defined on index sets $J$ and $K$ as in (3.1), and then replacing $\eta_{1}, \theta_{1}$ and 
$x_{i}$ with $\ln \eta_{1}, \ln \theta_{1}$ and $\ln x_{i}$ after simplification we get

$$
\begin{aligned}
& \widetilde{F}(J)=W_{J}\left(\widetilde{A}_{J}-\widetilde{G}_{J}\right), \\
& \widetilde{F}(K)=W_{K}\left(\widetilde{A}_{K}-\widetilde{G}_{K}\right) .
\end{aligned}
$$

As all the assumptions of Theorem 3.1 are satisfied, hence by substituting values of (6.16), (6.17) and (6.18) in (3.2) we get our required result.

The following corollary gives the relation between geometric and harmonic mean.

Corollary 6.5. Suppose all the assumptions of Theorem 6.4 hold. Then

$$
\begin{aligned}
& (i)\left(\frac{\widetilde{G}_{J \cup K}}{\widetilde{H}_{J \cup K}}\right)^{W_{J \cup K}} \geq\left(\frac{\widetilde{G}_{J}}{\widetilde{H}_{J}}\right)^{W_{J}}\left(\frac{\widetilde{G}_{K}}{\widetilde{H}_{K}}\right)^{W_{K}}, \\
& (i i) W_{J \cup K}\left(\frac{1}{\widetilde{H}_{J \cup K}}-\frac{1}{\widetilde{G}_{J \cup K}}\right) \geq W_{J}\left(\frac{1}{\widetilde{H}_{J}}-\frac{1}{\widetilde{G}_{J}}\right)+W_{K}\left(\frac{1}{\widetilde{H}_{K}}-\frac{1}{\widetilde{G}_{K}}\right) .
\end{aligned}
$$

Proof. (i)Consider the first assertion of Theorem 6.4 and then by substituting $\eta_{1} \rightarrow$ $\frac{1}{\eta_{1}}, \theta_{1} \rightarrow \frac{1}{\theta_{1}}, x_{i} \rightarrow \frac{1}{x_{i}}$ we get our required result. (ii) Consider the second assertion of Theorem 6.4 and then by substituting $\eta_{1} \rightarrow \frac{1}{\eta_{1}}, \theta_{1} \rightarrow \frac{1}{\theta_{1}}, x_{i} \rightarrow \frac{1}{x_{i}}$ we get our required result.

Now we define the relation between arithmetic and power mean.

Theorem 6.6. Suppose all the assumptions of Theorem 3.1 hold. Then

$$
\text { (i)For } r \leq 1, W_{J \cup K}\left(\widetilde{A}_{J \cup K}-\widetilde{M}_{J \cup K}^{[r]}\right) \geq W_{J}\left(\widetilde{A}_{J}-\widetilde{M}_{J}^{[r]}\right)+W_{K}\left(\widetilde{A}_{K}-\widetilde{M}_{K}^{[r]}\right) \text {. }
$$

(ii)For $r \geq 1$, the inequality (6.19) reversed.

Proof. Let $r \leq 1$. Applying the convex function $\zeta(x)=x^{\frac{1}{r}}$ for $x \in\left[\eta_{1}, \theta_{1}\right]$, to the functional $\widetilde{F}$ defined on index set $J \cup K$ as in relation (3.1).

$$
\begin{aligned}
& \widetilde{F}(J \cup K) \\
& =W_{J \cup K}\left(\eta_{1}^{\frac{1}{r}}+\theta_{1}^{\frac{1}{r}}-\frac{1}{W_{J \cup K}} \sum_{i \in J \cup K} w_{i} x_{i}^{\frac{1}{r}}-\left(\eta_{1}+\theta_{1}-\frac{1}{W_{J \cup K}} \sum_{i \in J \cup K} w_{i} x_{i}\right)^{\frac{1}{r}}\right),
\end{aligned}
$$

replacing $\eta_{1}, \theta_{1}$, and $x_{i}$ with $\eta_{1}^{r}, \theta_{1}^{r}$, and $x_{i}^{r}$ in equation (6.20) we get

$$
\begin{aligned}
& \widetilde{F}(J \cup K) \\
& =W_{J \cup K}\left(\eta_{1}+\theta_{1}-\frac{1}{W_{J \cup K}} \sum_{i \in J \cup K} w_{i} x_{i}-\left(\eta_{1}^{r}+\theta_{1}^{r}-\frac{1}{W_{J \cup K}} \sum_{i \in J \cup K} w_{i} x_{i}^{r}\right)^{\frac{1}{r}}\right) .
\end{aligned}
$$

Using (6.3) and (6.6) in equation (6.21) we get

$$
\widetilde{F}(J \cup K)=W_{J \cup K}\left(\widetilde{A}_{J \cup K}-\widetilde{M}_{J \cup K}^{[r]}\right) .
$$


Similarly, applying the convex function $f(x)=x^{\frac{1}{r}}$ for $x \in\left[\eta_{1}, \theta_{1}\right]$, to the functional $\widetilde{F}$ defined on index sets $J$ and $K$ as in (3.1), and then after simplification we get

$$
\begin{aligned}
& \widetilde{F}(J)=W_{J}\left(\widetilde{A}_{J}-\widetilde{M}_{J}^{[r]}\right), \\
& \widetilde{F}(K)=W_{K}\left(\widetilde{A}_{K}-\widetilde{M}_{K}^{[r]}\right) .
\end{aligned}
$$

As all the assumptions of Theorem 3.1 are satisfied, hence by substituting values of (6.22), (6.23) and (6.24) in (3.2) we get our required result. If $\mathrm{r} \geq 1$, then the function $\zeta(x)=x^{\frac{1}{r}}$ is concave, for $x \in\left[\eta_{1}, \theta_{1}\right]$ so the inequality (6.19) reversed.

The following corollary gives the relation between arithmetic and harmonic mean.

Corollary 6.7. Suppose all the assumptions of Theorem 6.6 hold. Then

$$
W_{J \cup K}\left(\widetilde{A}_{J \cup K}-\widetilde{H}_{J \cup K}\right) \geq W_{J}\left(\widetilde{A}_{J}-\widetilde{H}_{J}\right)+W_{K}\left(\widetilde{A}_{K}-\widetilde{H}_{K}\right) .
$$

Proof. As all the assumptions of Theorem 6.6 hold, hence for $r=-1$ and by using (iii) assertion of Remark 6.3 we get inequality (6.25).

Remark 6.8. It is easy to see that the assertion ( $i i)$ from Theorem 6.4 is also direct consequence of Theorem 6.6 by using second assertion of 6.3 .

Theorem 6.9. Suppose all the assumptions of Theorem 3.1 hold. Let $r, s \in \mathbb{R}$ and $r \leq s$.

(i)If $s \geq 0$, then

$$
\begin{aligned}
& W_{J \cup K}\left(\left(\widetilde{M}_{J \cup K}^{[s]}\right)^{s}-\left(\widetilde{M}_{J \cup K}^{[r]}\right)^{s}\right) \geq W_{J}\left(\left(\widetilde{M}_{J}^{[s]}\right)^{s}-\left(\widetilde{M}_{J}^{[r]}\right)^{s}\right)+ \\
& W_{K}\left(\left(\widetilde{M}_{K}^{[s]}\right)^{s}-\left(\widetilde{M}_{K}^{[r]}\right)^{s}\right) .
\end{aligned}
$$

(ii) If $s \leq 0$, then the inequality (6.26) reversed.

Proof. Consider the case $s \geq 0$. Applying the convex function $\zeta(x)=x^{\frac{s}{r}}$ for $x \in\left[\eta_{1}, \theta_{1}\right]$, to the functional $\widetilde{F}$ defined on index set $J \cup K$ as in relation (3.1)

$\widetilde{F}(J \cup K)$

$$
=W_{J \cup K}\left(\eta_{1}^{\frac{s}{r}}+\theta_{1}^{\frac{s}{r}}-\frac{1}{W_{J \cup K}} \sum_{i \in J \cup K} w_{i} x_{i}^{\frac{s}{r}}-\left(\eta_{1}+\theta_{1}-\frac{1}{W_{J \cup K}} \sum_{i \in J \cup K} w_{i} x_{i}\right)^{\frac{s}{r}}\right),
$$

by replacing $\eta_{1}, \theta_{1}$, and $x_{i}$ with $\eta_{1}^{r}, \theta_{1}^{r}$, and $x_{i}^{r}$ respectively in equation $(6.27)$ we get

$$
\begin{aligned}
& \widetilde{F}(J \cup K) \\
& =W_{J \cup K}\left(\eta_{1}^{s}+\theta_{1}^{s}-\frac{1}{W_{J \cup K}} \sum_{i \in J \cup K} w_{i} x_{i}^{s}-\left(\eta_{1}^{r}+\theta_{1}^{r}-\frac{1}{W_{J \cup K}} \sum_{i \in J \cup K} w_{i} x_{J}^{r}\right)^{\frac{s}{r}}\right) .
\end{aligned}
$$

Using (6.6) in (6.28) we get

$$
\widetilde{F}(J \cup K)=W_{J \cup K}\left(\left(\widetilde{M}_{J \cup K}^{[s]}\right)^{s}-\left(\widetilde{M}_{J \cup K}^{[r]}\right)^{s}\right) .
$$


Similarly, applying the convex function $\zeta(x)=x^{\frac{s}{r}}$ for $x \in\left[\eta_{1}, \theta_{1}\right]$, to the functional $\widetilde{F}$ defined on index sets $J$ and $K$ as in (3.1) and then after simplification we get

$$
\begin{aligned}
& \widetilde{F}(J)=W_{J}\left(\left(\widetilde{M}_{J}^{[s]}\right)^{s}-\left(\widetilde{M}_{J}^{[r]}\right)^{s}\right), \\
& \widetilde{F}(K)=W_{K}\left(\left(\widetilde{M}_{K}^{[s]}\right)^{s}-\left(\widetilde{M}_{K}^{[r]}\right)^{s}\right) .
\end{aligned}
$$

As all the assumptions of Theorem 3.1 are satisfied, hence by substituting values of $(6.29),(6.30)$ and $(6.31)$ in $(3.2)$ we get our required result. If $s \leq 0$, then the function $\zeta(x)=x^{\frac{s}{r}}$ is concave, so the inequality (6.26) reversed.

Remark 6.10. Let $J$ be a finite nonempty subset of positive integers and let $\zeta: I \longrightarrow$ $\mathbb{R}$ be a function. Let $x=\left\{x_{i}\right\}_{i \in J}$ be a real monotonic sequence such that $0<x_{i} \in I$ for all $i \in J$, and $w=\left\{w_{i}\right\}_{i \in J}$ be a real sequence such that $0<\frac{\sum_{i \in J} w_{i} x_{i}}{W_{J}} \in I$, where $W_{J}=\sum_{i \in J} w_{i}$. If we define

Arithmetic mean

$$
A_{J}=\frac{1}{W_{J}} \sum_{i \in J} w_{i} x_{i},
$$

Geometric mean

$$
G_{J}=\left(\prod_{i \in J} x_{i}^{w_{i}}\right)^{\frac{1}{W_{J}}}
$$

Harmonic mean

$$
H_{J}=\left(\frac{1}{W_{J}} \sum_{i \in J} w_{i} x_{i}^{-1}\right)^{-1}
$$

Power mean

$$
M_{J}^{[r]}=\left(\frac{1}{W_{J}} \sum_{i \in J} w_{i} x_{i}^{r}\right)^{r},
$$

then the analogues assertions of all above results can be formulated on these means for using weights which satisfying conditions of Theorem 2.1.

\section{Conclusion}

Vasić et. al. in [38] and Matković et. al. in [28] used index set functions to give refinements of Jensens and Jensen-Mercer inequalities. It is reasonable to inquire that whether it is possible to give refinements of Jensens and Jensen-Mercer inequalities with Jensen Steffensens conditions using an index set functions. The present article aimed to answer this query. For this purpose we constructed some index set functions and used Jensen Steffensens conditions to give generalization followed by the refinement of Jensens and Jensen-Mercer inequalities. Furthermore, we obtained some integral and discrete nature refinements of Jensen-Mercer inequality. These refinements obtained as an improvement of the upper bound of the Jensens difference given in [32]. Based on our findings, we gave some new bounds of some important inequalities used in information theory, and also generalized the relations among means. 


\section{REFERENCES}

[1] S. Abramovich, M. Klaričić Bakula, M. Matić and J. E. Pečarić, A variant of JensenSteffensen's inequality and quasi-arithmetic means, J. Math. Anal. Appl., 307 (2005), 370386.

[2] M. M. Ali, A. R. Khan, I. U. Khan, S Saadi, Improvement of Jensen and Levinson Type Inequalities for Functions with Nondecreasing Increments, Glob. J. Pure Appl. Math., 15, (6) (2019) 945-970.

[3] M. M. Ali, A. R. Khan, Generalized integral Mercer's inequality and integral Means, JIASF 10, (1) (2019), 60-67.

[4] M. K. Bakula, J. Pečarić, J. Perić, On the converse Jensen inequality, Appl. Math. Comput., 218 (11) (2012), 6566-6575.

[5] P. S. Bullen, D. S. Mitrinović, and P. M. Vasić, Means and their inequalities, Reidel, Dordrecht, 1988.

[6] P. S. Bullen, Rado's inequality, Aequationes Math., 6 (2-3) (1971), 149-156.

[7] W. S. Cheung, A. Matković and J. E. Pečarić, A variant of Jessen's inequality and generalized means, J. Inequal. Pure Appl. Math., 7 (1) (2006), Article 10, 1-8.

[8] S. S. Dragomir, A new refinement of Jensen's inequality in linear spaces with applications, Math. Comput. Model., 52 (9-10) (2010), 1497-1505.

[9] S. S. Dragomir, A refinement of Jensen's inequality with applications for f-divergence measures, Taiwanese J. Math., 14 (1) (2010), 153-164.

[10] S. S. Dragomir, On Hadamard's inequality for the convex mappings defined on a ball in the space and applications, Math. Inequal. Appl., 3 (2) (2000), 177-187.

[11] S. S. Dragomir, Some refinements of Jensens inequality, J. Math. Anal. Appl., 168 (2) (1992), 518-522.

[12] L. Horváth, D. PeČarić And J. PeČArić, Estimation of $f$ - and Rényi divergences by using a cyclic refinement of the Jensen's inequality, Bull. Malays. Math. Sci. Soc., 42 (2019), 933-946.

[13] L. HoRváth, A parameter-dependent refinement of the discrete Jensen's inequality for convex and mid-convex functions, J. Inequal. Appl., 2011 (1) (2011), Article 26.

[14] S. Hussain And J. PEČARIĆ, An improvement of Jensen's inequality with some applications, Asian-European J. Math., 2 (1) (2009), 85-94.

[15] J. L. W. V. JEnsen, Sur les fonctions convexes et les ingalits entre les valeurs moyennes, (French) Acta Math., 30 (1) (1906), 175-193.

[16] J. L. W. V. Jensen, Om konvexe funktioner og uligheder mellem Middelvaerdier, (German) Nyt. Tidsskrift Math., 16B (1905), 49-69.

[17] A. R. Khan, I. U. Khan, An extension of Jensen Mercer inequality for functions with non decresing increments, JIASF, 10, (4) (2019).

[18] A. R. Khan, I. U. Khan, Some remarks on Niezgoda's extension of Jensen Mercer inequality, Adv. Inequal. Appl., 2016, Article ID 12, (2016).

[19] A. R. Khan, I. U. Khan, And S. S. A. RamJI, Generalizations and refinements of Niezgoda's extension of Jensen-Mercer inequality with applications, J. Math. Inequal., in press.

[20] M. A. Khan, A. R. Khan, And J. E. PeČARIĆ, On the refinements of Jensen-Mercer's inequality, Rev. Anal. Numér. Théor. Approx., 41 (1) (2012), 62-81.

[21] A. R. Khan, S. SAAdi, Generalizations and refinements of Niezgoda inequality for similarly separable vectors with applications, FILOMAT, in press.

[22] A. R KHAN, S. SAADI, Generalized Jensen Mercer inequality for functions with nondecreasing increments, Abstract Appl. Anal., 2016, Article ID 5231476, (2016), 1-12.

[23] A. R Khan, J. E. PeČArić and M. R. Lipanović, Exponential Convexity for Jensen type Inequalities, J. Math. Inequal. , 7, (3) (2013), 313-335.

[24] M. A. Khan, A. R. Khan And J. E. PeČArić, On the refinements of Jensen-Mercer's inequality, Rev. Anal. Numér. Théor. Approx., 41 (1) (2012), 62-81.

[25] P. Lah, M. Rabaric, Converse of Jensen's inequality for convex functions, Univ. Beograd Publ. Elektrotehn. Fak. Ser. Mat. Fiz., No. 412 - 460 (1973), $201-205$.

[26] A. LESNE, Shannon entropy: a rigorous notion at the crossroads between probability, information theory, dynamical system and statistical physics, Math. Struct. Comput. Sci., 24 (3) (2014). 
[27] A. Matković, J. E. Pečarić, J. Perić, A refinement of the Jensen-Mercer's inequality and generalization on convex hulls in $\mathbb{R}^{k}$, J. Math. Inequal, 9 (4) (2015), 1093-1114.

[28] A. Matković, And J. E. PeČARić, Refinements of the Jensen-Mercer's inequality for index set functions with applications, Rev, Anal. Numér. Théor. Approx., 35 (1) (2006), 71-82.

[29] A. M. Mercer, A variant of Jensen's inequality, J. Inequal. Pure Appl. Math., 4 (4) (2003), Article 73.

[30] D. S. Mitrinović, J. E. PeČarić And A. M. Fink, Classical and new inequalities in analysis, Kluwer Academic Publishers Group, Dordrecht, 1993.

[31] D. S. Mitrinović, Analytic inequalities, Springer-Verlag, New York-Berlin, 1970.

[32] J. PEČARIC, J. PERIĆ, Remarks on the paper Jensens inequality and new entropy bounds by S. Simic, Math. Inequal. Appl., 6 (4) (2012), 631-636.

[33] J. E. PeČarić, F. Proschan And Y. L. Tong, Convex functions, partial orderings and statistical applications, Academic Press, New York, 1992.

[34] J. Rooin, Some refinements of discrete Jensen's inequality and some of its applications, Nonlinear Functional Anal. Appl., 12 (1) (2007), 107-118.

[35] S. Simić, Jensen's inequality and new entropy bounds, Appl. Math. Lett., 22 (2019), 12621265 .

[36] B. Simon, Convexity: an analytic viewpoint, Cambridge University Press, Cambridge, 2011.

[37] J. F. Steffensen, On certain inequalities and methods of approximation, J. Inst. Actuaries, (3) (1919), 274-279.

[38] P. M. VASiĆ AND Ž. MiJalković, On an index set function connected with Jensen inequality, Univ. Beograd. Publ. Elektrotehn. Fak. Ser. Mat. Fiz., 544-576 (1976), 110-112.

[39] L. C. WANG, X. F. MA, AND L. H. LIU, A note on some new refinements of Jensen's inequality for convex functions, J. Ineq. Pure Appl. Math., 10 (2) (2009), Article 48.

[40] G. Zabandan AND A. Kilicman, A new version of Jensen's inequality and related results, J. Inequal. Appl., 2012 (2012), Article 238.

FAIZA RUBAB

Department of Mathematics, University of Karachi, University Road, Karachi 75270 , PAKISTAN.

E-mail address: faizarizwan12@gmail.com

HIRA NABI

Department of Mathematics, University of Karachi, University Road, Karachi 75270 , PAKISTAN.

E-mail address: hira_nabi@yahoo.com

ASIF RAZA KHAN

Department of Mathematics, University of Karachi, University Road, Karachi 75270 , PAKISTAN.

E-mail address: asifrk@uok.pk 